This is an accepted manuscript of an article published by Taylor \& Francis in International Journal of

General Systems on 5 Mar 2019, available online: https://doi.org/10.1080/03081079.2019.1586684

\title{
Curve-based monotonicity: a generalization of directional monotonicity
}

Antonio F. Roldán-López-de-Hierro ${ }^{a}$, Mikel Sesma-Sara ${ }^{\text {b,c }}$, Jana Špirkovád, Julio Lafuente $^{\mathrm{b}}$, Ana Pradera ${ }^{\mathrm{e}}$, Radko Mesiar ${ }^{\mathrm{f}, \mathrm{g}}$ and Humberto Bustince ${ }^{\mathrm{b}, \mathrm{c}}$

a Department of Mathematics Education, Faculty of Science Education, Campus de Cartuja,

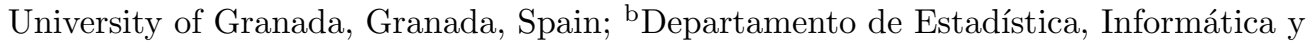
Matemáticas, Universidad Pública de Navarra, Campus Arrosadia s/n, 31006, Pamplona, Spain; ${ }^{\mathrm{C}}$ Institute of Smart Cities, Universidad Pública de Navarra, 31006, Pamplona, Spain; ${ }^{\mathrm{d}}$ Department of Quantitative Methods and Information Systems, Faculty of Economics,

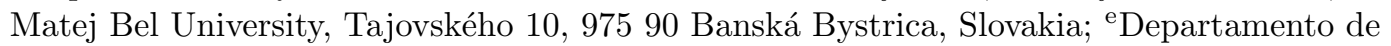
Ciencias de la Computación, Arquitectura de Computadores, Lenguajes y Sistemas Informáticos y Estadística e Investigación Operativa, Universidad Rey Juan Carlos, Móstoles, Madrid 28933, Spain; ${ }^{\mathrm{f}}$ Department of Mathematics and Descriptive Geometry, Faculty of Civil Engineering, Slovak University of Technology, Radlinského 11, Bratislava, Slovakia; ${ }^{\mathrm{g}}$ Institute for Research and Applications of Fuzzy Modelling, University of Ostrava, 30. dubna 22, Ostrava 1, Czech Republic

\section{ARTICLE HISTORY}

Compiled February 19, 2019

\begin{abstract}
In this work we propose a generalization of the notion of directional monotonicity. Instead of considering increasingness or decreasingness along rays, we allow more general paths defined by curves in the $n$-dimensional space. These considerations lead us to the notion of $\alpha$-monotonicity, where $\alpha$ is the corresponding curve. We study several theoretical properties of $\alpha$-monotonicity and relate it to other notions of monotonicity, such as weak monotonicity and directional monotonicity.
\end{abstract}

\section{KEYWORDS}

Fusion function; weak monotonicity; directional monotonicity; curve-based monotonicity

\section{Introduction}

Aggregation functions (Calvo et al. 2002; Grabisch et al. 2009), in general, and means, in particular (Beliakov, Bustince and Calvo 2016), are critical tools to deal with problems based on information aggregation (Bustince et al. 2012; Jurio et al. 2013; Melin, Bravo and Castillo 2008; Su, Xia and Chen 2011; Ye 2013). A key property in the definition of such functions is monotonicity and, more specifically, monotone increasingness (Beliakov 2005). However, in some applications, the aggregation of information is performed using tools which do not satisfy monotonicity, examples being:

(1) In image preprocessing, in order to efficiently identify snow in a given image, 
which is relevant to approximate the existing reserves of water (Hall, Riggs and Salomonson 1995; Soria-Frisch 2009; Storvold and Malnes 2004). There exist algorithms to solve this problem which make use of the mode operator to aggregate. However, since the mode is not uniquely defined as a function (as it may be multivalued), it does not make sense to speak of monotonicity in this case. Furthermore, even if the mode is understood as a function, it is not monotone.

(2) Applications where penalty-based aggregation functions are used (Bustince et al. 2014). Any averaging aggregation function (i.e. any aggregation function which is bounded by the maximum and the minimum of its inputs) can be recovered from an appropriate quasi-convex function, called penalty function, through a minimization procedure. However, the converse is not true. In general, given a penalty function, the corresponding minimization procedure leads to a function which, although always averaging, needs not be an aggregation function, since monotonicity is not ensured.

In recent years, the relaxation of monotonicity has become a trend in the theory of aggregation functions (Mesiar, Kolesárová and Stupňanová 2018) and there exists a growing interest on analyzing different types of monotonicity which can cover some of the previously discussed situations (Wilkin and Beliakov 2015; Bustince et al. 2015, 2018; Sesma-Sara et al. In press). Two of these notions of monotonicity are specially relevant:

(1) The idea of weak monotonicity (Wilkin and Beliakov 2015; Beliakov, Calvo and Wilkin 2015), which, for a function of $n$ variables, considers increasingness or decreasingness only along the ray defined by the vector $(1, \ldots, 1)$. It turns out that this notion allows to cover some relevant statistical operators which are not monotone, as, for instance, the mode.

(2) The idea of directional monotonicity (Bustince et al. 2015), which generalizes weak monotonicity considering increasingness or decreasingness along a ray which can be defined by any vector. When a function is directionally increasing with respect to a set of vectors that form a cone, it is called cone-monotone (Beliakov, Calvo and Wilkin 2014). This ideas have led to the notion of preaggregation functions, which is a function fulfilling the same boundary conditions as an aggregation function but which is just directionally increasing along some ray. Pre-aggregation functions have shown themselves a very powerful tool in classification problems (Lucca et al. 2016).

The objective of this work is to consider increasingness or decreasingness along paths more general than rays.

In order to define this generalized form of directional monotonicity, we are going to consider increasingness or decreasingness along a curve (path) $\alpha$. For this reason, we are going to call the new concept $\alpha$-monotonicity. Note that $\alpha$-monotonicity extends directional monotonicity, as rays (straight lines) are particular instances of paths.

The consideration of monotonicity along paths leads to some problems from a theoretical point of view. In particular, the possibility of considering closed curves must be carefully analyzed, as well as the regularity conditions ensuring some sort of composition between $\alpha$ - and $\beta$-monotonicity. These problems do not arise in the case of directional monotonicity, and they are not trivial. Furthermore, they are specially relevant for possible applications of our developments that we intend to discuss in future works, specially those in image processing, as edges can be described in terms of closed paths. For this reason, in this work we focus on the theoretical analysis of 
the new concept to solve these difficulties and we leave for a future work the study of applications.

The structure of this work is as follows. We start with some preliminaries and, in particular, we review the classical notion of monotonicity. Section 3 is devoted to the study of the new notion of monotonicity. Section 4 discusses several properties of $\alpha$-monotone functions. In Section 5 we comment some relevant aspects of the new concept from an algebraical and an analytical point of view. In Section 6 we study idempotency and averageness for this new type of functions. We finish with some concluding remarks and references.

\section{Preliminaries}

\subsection{Basic notations and concepts}

Throughout this manuscript, let $n \in \mathbb{N}$ be such that $n \geq 2$. Points (or vectors) in $\mathbb{R}^{n}$ will be represented in boldface, that is, $\mathbf{x}=\left(x_{1}, x_{2}, \ldots, x_{n}\right) \in \mathbb{R}^{n}$, where $x_{1}, x_{2}, \ldots, x_{n} \in \mathbb{R}$. Let $\mathbf{0}=(0,0, \ldots, 0)$ and $\mathbf{1}=(1,1, \ldots, 1)$. We use the standard partial order in $\mathbb{R}^{n}$, i.e., we say that $\mathbf{x} \leq \mathbf{y}$ if $x_{i} \leq y_{i}$ for every $i \in\{1, \ldots, n\}$.

Given a function $f: D \rightarrow \mathbb{R}$ defined on a nonempty subset $D \subseteq \mathbb{R}$, we say that $f$ is increasing if $f(t) \leq f(s)$ for all $t, s \in D$ such that $t \leq s$. Similarly, $f$ is decreasing if $f(t) \geq f(s)$ for all $t, s \in D$ such that $t \leq s$.

We will refer to functions $F:[0,1]^{n} \rightarrow[0,1]$ as fusion functions. A fusion function $F:[0,1]^{n} \rightarrow[0,1]$ is:

- idempotent if $F(t, t, \ldots, t)=t$ for all $t \in[0,1]$;

- an averaging function if $\min (\mathbf{x}) \leq F(\mathbf{x}) \leq \max (\mathbf{x})$ for all $\mathbf{x} \in[0,1]^{n}$.

Note that any averaging fusion function is idempotent.

A ( $n$-dimensional) curve on $\mathbb{R}^{n}$ is a mapping $\alpha: I_{\alpha} \rightarrow \mathbb{R}^{n}$ where the domain $I_{\alpha}$ is a nonempty subinterval of $\mathbb{R}$. For our purposes, throughout this manuscript, we will only consider curves defined on intervals (non reduced to a singleton) of nonnegative real numbers (that is, $I_{\alpha} \subseteq[0, \infty[$ ) and such that

$$
0 \in I_{\alpha} \quad \text { and } \quad \alpha(0)=\mathbf{0} .
$$

Hence, there are only three cases for this domain: $[0, \infty[$ (if it is unbounded), $[0, \theta]$ (if it is bounded and closed on $[0, \infty[$ ) or $[0, \theta[$ (if it is bounded and open on $[0, \infty[$ ), where $\theta \in] 0, \infty[$. Most of the properties we show in this paper do not depend on the character of the domain, that is, the same property holds if the domain is open or closed on $[0, \infty[$. However, there also exist some properties that are based on the closure of the domain (see Subsection 5.1).

We denote by $\alpha_{1}, \alpha_{2}, \ldots, \alpha_{n}: I_{\alpha} \rightarrow \mathbb{R}$ the components of $\alpha$, that is,

$$
\alpha(t)=\left(\alpha_{1}(t), \ldots, \alpha_{n}(t)\right) \text { for all } t \in I_{\alpha} .
$$

We also denote by $\alpha_{\mathbf{0}}$ the curve $\alpha_{\mathbf{0}}: I_{\alpha_{\mathbf{0}}} \rightarrow \mathbb{R}^{n}$ given by $\alpha_{\mathbf{0}}(t)=\mathbf{0}$ for all $t \in I_{\alpha_{\mathbf{0}}}$ (where $I_{\alpha_{0}}$ is an interval as above). 
We employ $\|\cdot\|_{1},\|\cdot\|_{2}$ and $\|\cdot\|_{\infty}$ to denote the usual norms in $\mathbb{R}^{n}$; given $\mathbf{x} \in \mathbb{R}^{n}$,

$$
\|\mathbf{x}\|_{1}=\sum_{i=1}^{n}\left|x_{i}\right|, \quad\|\mathbf{x}\|_{2}=\left(\sum_{i=1}^{n}\left|x_{i}\right|^{2}\right)^{1 / 2} \quad \text { and } \quad\|\mathbf{x}\|_{\infty}=\max _{1 \leq i \leq n}\left|x_{i}\right| .
$$

If $\|\cdot\|$ is a norm on $\mathbb{R}^{n}$, the ball of center $\mathbf{x} \in \mathbb{R}^{n}$ and radius $r>0$ is $B(\mathbf{x}, r)=$ $\left\{\mathbf{y} \in \mathbb{R}^{n}:\|\mathbf{x}-\mathbf{y}\| \leq r\right\}$.

Remark 2.1. Note that if $\mathbf{x} \in[0,1]^{n}$ and $\mathbf{y} \in \mathbb{R}^{n}$ are such that $\mathbf{x}+\mathbf{y} \in[0,1]^{n}$, then $\|\mathbf{y}\|_{\infty} \leq 1$. In particular, if $\mathbf{x} \in[0,1]^{n}$ and $t \in I_{\alpha}$ are such that $\mathbf{x}+\alpha(t) \in[0,1]^{n}$, then

$$
\max \left(\left|\alpha_{1}(t)\right|, \ldots,\left|\alpha_{n}(t)\right|\right) \leq 1
$$

\subsection{Monotone fusion functions}

This work mainly deals with a generalization of the notion of monotonicity. For this reason, we recall here the well-known classical concepts of monotone increasing and monotone decreasing function.

Definition 2.2. A function $F:[0,1]^{n} \rightarrow[0,1]$ is monotone increasing (respectively, monotone decreasing) if

$$
F\left(x_{1}, \ldots, x_{n}\right) \leq F\left(y_{1}, \ldots, y_{n}\right)\left(\text { respectively, } F\left(x_{1}, \ldots, x_{n}\right) \geq F\left(y_{1}, \ldots, y_{n}\right)\right)
$$

for every $x_{1}, \ldots, x_{n}, y_{1}, \ldots, y_{n} \in[0,1]$ such that $x_{i} \leq y_{i}$ for every $i \in\{1, \ldots, n\}$.

For the sake of simplicity, monotone increasing and monotone decreasing functions are said to be increasing and decreasing, respectively.

Definition 2.3. A function which is increasing or decreasing is called a monotone function.

In this work we usually refer to increasing functions, but most of the results can be easily adapted to the case of decreasing functions.

Example 2.4. (1) Every constant function is both increasing and decreasing. In fact, constant functions are the only functions which are at the same time increasing and decreasing.

(2) A differentiable function $F:[0,1]^{n} \rightarrow[0,1]$ is increasing if and only if

$$
\frac{\partial F}{\partial y_{i}}\left(y_{1}, \ldots, y_{n}\right) \geq 0
$$

for every $y_{1}, \ldots, y_{n} \in[0,1]$ (with the corresponding adjustments in the boundary of $\left.[0,1]^{n}\right)$. Note that this is equivalent to requiring that, for any $\mathbf{h} \in[0,1]^{n}$, the inequality

$$
\nabla F\left(y_{1}, \ldots, y_{n}\right) \cdot \mathbf{h} \geq 0
$$


holds for every $y_{1}, \ldots, y_{n} \in[0,1]$, where $\nabla F$ denotes the gradient of $F$ :

$$
\nabla F=\left(\frac{\partial F}{\partial y_{1}}, \ldots, \frac{\partial F}{\partial y_{n}}\right)
$$

The usual definition of aggregation function (Calvo et al. 2002; Gómez and Montero 2004; Grabisch et al. 2009) makes use of the notion of monotonicity, as follows.

Definition 2.5. An aggregation function is a fusion function $A:[0,1]^{n} \rightarrow[0,1]$ such that:

(1) $A(0, \ldots, 0)=0$ and $A(1, \ldots, 1)=1$;

(2) $A$ is increasing.

\subsection{Extensions of monotonicity: weak monotonicity and directional monotonicity}

Imposing monotonicity might be too restrictive for some specific applications. As an example, we analyze the case of the mode. We define the mode of a given set of inputs as that input which appears most often. If there are more than one candidate inputs, we choose the smallest one.

If $n \geq 2$, the mode function is monotone if and only if $n=2$, and in this case it is increasing. In fact, if $n=2$, clearly $\bmod (\mathbf{x})=\min \left(x_{1}, x_{2}\right)$, and so $\mathbf{x} \leq \mathbf{y}$ implies $\bmod (\mathbf{x}) \leq \bmod (\mathbf{y})$.

To see that for $n \geq 3$ it is not monotone it suffices to suppose that $n=3$. If it were monotone, it should be increasing, but this is not the case by considering, for instance, $\mathbf{x}=(0.4,0.6,0.6)<\mathbf{y}=(0.4,0.6,0.7)$ and $\bmod (\mathbf{x})=0.6>0.4=\bmod (\mathbf{y})$.

However, if all the inputs of the mode are increased by the same amount, then the mode itself increases. These considerations led to the introduction of the notion of weak monotonicity (Wilkin and Beliakov 2015).

Definition 2.6 (Wilkin and Beliakov (2015)). A function $F:[0,1]^{n} \rightarrow[0,1]$ is said to be weakly monotone increasing (respectively, weakly monotone decreasing) if the inequality

$$
\begin{aligned}
F\left(x_{1}+h, \ldots, x_{n}+h\right) \geq & F\left(x_{1}, \ldots, x_{n}\right) \\
& \left(\text { respectively }, F\left(x_{1}+h, \ldots, x_{n}+h\right) \leq F\left(x_{1}, \ldots, x_{n}\right)\right)
\end{aligned}
$$

holds for every $x_{1}, \ldots, x_{n}, h \in[0,1]$ such that $x_{i}+h \leq 1, i \in\{1, \ldots, n\}$.

A function $F$ is said to be weakly monotone when it is weakly monotone increasing or weakly monotone decreasing.

We have the following straightforward result.

Proposition 2.7. Every increasing (respectively, decreasing) fusion function is also weak monotone increasing (respectively, decreasing).

However, the converse is false because a weakly monotone function does not need to be increasing (or decreasing), as the case of the mode shows.

From a geometrical point of view, weak monotonicity corresponds to considering monotonicity along the fixed ray defined by the vector $\mathbf{1}=(1, \ldots, 1)$ starting at any 
point of the domain of the function.

Weak monotonicity was further extended into the notion of directional monotonicity in the following way.

Definition 2.8 (Bustince et al. (2015)). Let $\mathbf{r}$ be a real vector $(\mathbf{r} \neq \mathbf{0})$. A fusion function $F:[0,1]^{n} \rightarrow[0,1]$ is $\mathbf{r}$-increasing (respectively, $\mathbf{r}$-decreasing) if for every $\mathbf{x} \in[0,1]^{n}$ and for every $c>0$ such that $\mathbf{x}+c \mathbf{r} \in[0,1]^{n}$ it holds that:

$$
F(\mathbf{x}+c \mathbf{r}) \geq F(\mathbf{x}) \quad(\text { respectively, } F(\mathbf{x}+c \mathbf{r}) \leq F(\mathbf{x})) .
$$

A function $F$ which is $\mathbf{r}$-increasing or $\mathbf{r}$-decreasing is said to be a directionally monotone function.

Definition 2.9 (Beliakov, Calvo and Wilkin (2014)). Let $C \subset \mathbb{R}^{n}$ be a nonempty cone. A fusion function $F:[0,1]^{n} \rightarrow[0,1]$ is cone-increasing (respectively, conedecreasing) if it is $\mathbf{r}$-increasing (respectively, $\mathbf{r}$-decreasing) for every $\mathbf{r} \in C$.

A function $F$ which is cone-increasing or cone-decreasing is said to be a conemonotone.

Example 2.10. - Fuzzy implication functions (see Bustince, Burillo and Soria 2003) are $(-1,1)$-increasing functions. This implies that many other functions, which are widely used in applications and which can be obtained from implication functions, are also directionally increasing. This is the case, for instance, of some subsethood measures (see Bustince, Pagola and Barrenechea 2007).

- Many functions used for comparison of data are also directionally increasing. In particular, this is the case of those based on component-wise comparison by means of the Euclidean distance $|x-y|$, as for restricted equivalence functions (see Bustince et al. 2006; Sesma-Sara et al. 2018).

- Weakly increasing (decreasing) functions in the sense of Definition 2.6 are particular cases of directionally increasing (decreasing) functions in the sense of Definition 2.8 , by considering the vector $\mathbf{1}=(1, \ldots, 1)$. But there are many examples of directionally increasing functions which are not weakly increasing, as, for instance, implication functions (Bustince et al. 2015).

Directional monotonicity combined with appropriate boundary conditions leads to the notion of pre-aggregation function (Lucca et al. 2016).

\section{Monotonicity along curves}

All the notions of monotonicity that we have considered can be understood, from a geometrical point of view, as increasingness or decreasingness along one or more appropriate directions. Following this interpretation, this definition amounts to consider increasingness or decreasingness along a specific type of curves, namely, straight lines. So, in the same way, as the notion of weak monotonicity (monotonicity along the line defined by the vector $(1, \ldots, 1))$ has been extended to consider $\mathbf{r}$-monotonicity (i.e., monotonicity along the line defined by the vector $\mathbf{r}$, with $\mathbf{r}$ any real vector), it is natural to go one step beyond and to consider monotonicity along more general types of curves.

In order to carry this generalization out, we first introduce some notions. Recall that, throughout this manuscript, a curve is a mapping $\alpha: I_{\alpha} \rightarrow \mathbb{R}^{n}$ such that $\alpha(0)=\mathbf{0}$, 
where the domain $I_{\alpha}$ is $[0, \infty[,[0, \theta]$ or $[0, \theta[$ (for some $\theta \in] 0, \infty[$ ).

\subsection{Definition and first examples}

We use a curve to determine the direction along which we consider increasingness or decreasingness for a given function. In particular, given a curve $\alpha$, we are going to define $\alpha$-monotonicity (or monotonicity along the curve $\alpha$ ) as follows.

Definition 3.1. Let $F:[0,1]^{n} \rightarrow[0,1]$ be a fusion function and let $\alpha: I_{\alpha} \rightarrow \mathbb{R}^{n}$ be a curve (recall that $\alpha(0)=\mathbf{0})$. We say that $F$ is $\alpha$-increasing if

$$
\begin{array}{ll}
F(\mathbf{x}+\alpha(t)) \geq F(\mathbf{x}) \quad & \text { for all } \mathbf{x} \in[0,1]^{n} \text { and all } t \in I_{\alpha} \backslash\{0\} \\
& \text { such that } \left.\left.\mathbf{x}+\alpha(s) \in[0,1]^{n} \text { for all } s \in\right] 0, t\right] .
\end{array}
$$

Analogously, $F$ is $\alpha$-decreasing if

$$
F(\mathbf{x}+\alpha(t)) \leq F(\mathbf{x})
$$

for all $\mathbf{x} \in[0,1]^{n}$ and all $t \in I_{\alpha} \backslash\{0\}$ such that $\mathbf{x}+\alpha(s) \in[0,1]^{n}$ for all $\left.\left.s \in\right] 0, t\right]$.

Similarly, $F$ is $\alpha$-monotone if $F$ is $\alpha$-increasing or $\alpha$-decreasing, and $F$ is $\alpha$-constant if $F$ is at the same time $\alpha$-increasing and $\alpha$-decreasing.

Remark 3.2. Note that the fact that a fusion function $F$ is $\alpha$-increasing, for a certain curve $\alpha$, does not mean that the function increases along the graph of such curve, nor that it increases along the directions that join each point $\mathbf{x}$ with $\mathbf{x}+\alpha(t)$. Rather, $F$ being $\alpha$-increasing refers to the property of non-decreasingness in the values of $F$ when evaluating the points across which, starting from a fixed $\mathbf{x} \in[0,1]^{n}$, the curve $\alpha$ goes. The mentioned non-decreasingness is valued with respect to $F(\mathbf{x})$. Namely, it could be stated that a function $F$ is $\alpha$-increasing if, for all $\mathbf{x} \in[0,1]^{n}$, it holds that $F(\mathbf{x}+\alpha(t)) \geq F(\mathbf{x})$ for all $t \in I_{\alpha} \backslash\{0\}$, provided that no point $\mathbf{x}+\alpha(s)$ has left the unit hypercube $[0,1]^{n}$ for $s \leq t$.

The cases in which the curve leaves the unit hypercube and the differences between this notion and monotonicity along a curve are discussed in Sections 3.2 and 3.3, respectively.

Example 3.3. (1) Let us consider the degenerated curve $\alpha_{\mathbf{0}}(t)=\mathbf{0}$ for each $t \in I_{\alpha_{0}}$. Then every fusion function $F:[0,1]^{n} \rightarrow[0,1]$ is $\alpha_{0}$-increasing and $\alpha_{0}$-decreasing at the same time (that is, it is $\alpha_{0}$-constant).

(2) Weak monotonicity can be recovered as a specific case of $\alpha$-monotonicity, just taking $\alpha(t)=(t, \ldots, t)$ for all $t \in I_{\alpha}=[0,1]$.

(3) More generally, $\mathbf{r}$-monotonicity is recovered taking $I_{\alpha}=[0, \infty[$ and $\alpha(t)=t \mathbf{r}$ for all $t \in[0, \infty[$.

(4) Let us assume that $I_{\alpha}=[0,1]$, that $\alpha$ is continuous and $\lim _{t \rightarrow 1^{-}} \alpha(t)=\mathbf{0}$. This context corresponds to the case of a closed curve. If $F$ is an $\alpha$-increasing fusion function and for some $\mathbf{x} \in[0,1]^{n}$ we have that $\mathbf{x}+\alpha(t) \in[0,1]^{n}$ for all $t \in I_{\alpha}$, then $F(\mathbf{x})=F(\mathbf{y})$ for all $\mathbf{y}=\mathbf{x}+\alpha(t)$, where $t \in I_{\alpha}$, that is, $F$ is constant along the path $s \mapsto \mathbf{x}+\alpha(s)$. 
Example 3.4. Consider the arithmetic mean

$$
M\left(x_{1}, \ldots, x_{n}\right)=\frac{x_{1}+\cdots+x_{n}}{n} \text { for all } x_{1}, x_{2}, \ldots, x_{n} \in[0,1] .
$$

Then $M$ is $\alpha$-increasing for every curve $\alpha$ such that

$$
\sum_{i=1}^{n} \alpha_{i}(t) \geq 0 \quad \text { for all } t \in I_{\alpha} .
$$

Analogously, it is $\alpha$-decreasing for every $\alpha$ such that

$$
\sum_{i=1}^{n} \alpha_{i}(t) \leq 0 \quad \text { for each } t \in I_{\alpha}
$$

If we combine these two facts we deduce that, if we consider a curve $\alpha$ such that

$$
\sum_{i=1}^{n} \alpha_{i}(t)=0 \quad \text { for every } t \in I_{\alpha},
$$

it follows that $M$ is $\alpha$-constant.

Concretely, for the case $n=3$, let us consider the curve $\alpha:[0,1] \rightarrow \mathbb{R}^{3}$ given by

$$
\alpha(t)=\left(\frac{t}{2} \cos (6 \pi t), \frac{t}{2} \sin (6 \pi t),-\frac{t}{2}(\cos (6 \pi t)+\sin (6 \pi t))\right),
$$

whose graph is depicted in Figure 1. This curve satisfies $\alpha_{1}(t)+\alpha_{2}(t)+\alpha_{3}(t)=0$ for all $t \in[0,1]$ and, thus, the arithmetic mean $M\left(x_{1}, x_{2}, x_{3}\right)=\frac{x_{1}+x_{2}+x_{3}}{3}$ is $\alpha$-constant for the curve $\alpha$ given in eq. (3).

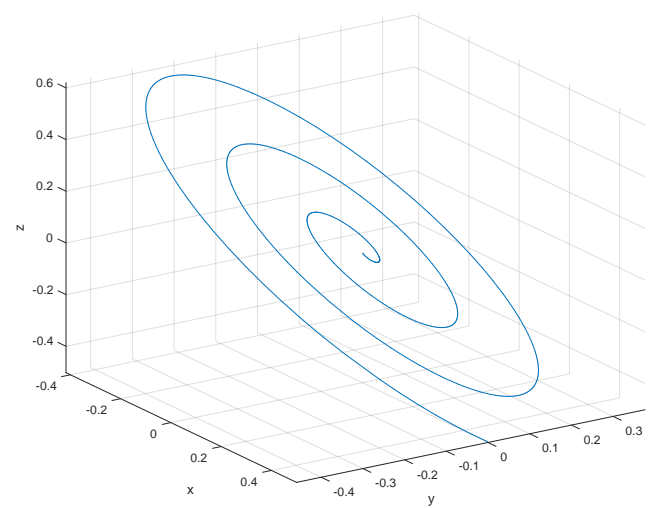

Figure 1. Graph of the curve $\alpha:[0,1] \rightarrow \mathbb{R}^{3}$ given by eq. (3).

Example 3.5. Let $C>2$ and define the function $F:[0,1]^{2} \rightarrow \mathbb{R}$ given by

$$
F(x, y)=0.5+\frac{x^{2}-y^{2}}{C} \text { for all } x, y \in[0,1] \text {. }
$$


Then $F$ is a fusion function. It can be proved that if $F$ is $\mathbf{r}$-increasing for some vector $\mathbf{r}=\left(r_{1}, r_{2}\right) \in \mathbb{R}^{2} \backslash\{\mathbf{0}\}$, then $r_{1} \geq 0 \geq r_{2}$. Nevertheless, $F$ is increasing along curves whose components do not satisfy such inequalities. For instance, let $\alpha:\left[0, \infty\left[\rightarrow \mathbb{R}^{2}\right.\right.$ be the curve defined as

$$
\alpha(t)=(\sqrt{3 t}, \sin t) \quad \text { for all } t \in\left[0, \frac{1}{3}\right] .
$$

If $\mathbf{x}=(x, y) \in[0,1]^{2}$ and $t>0$ are such that $\mathbf{x}+\alpha(t) \in[0,1]^{2}$, then

$$
0<\sqrt{3 t} \leq x+\sqrt{3 t}=x+\alpha_{1}(t) \in[0,1]
$$

and this implies that $\sin t>0$. Therefore

$$
\begin{aligned}
F(\mathbf{x}+\alpha(t))-F(\mathbf{x}) & =0.5+\frac{(x+\sqrt{3 t})^{2}-(y+\sin t)^{2}}{C}-\left(0.5+\frac{x^{2}-y^{2}}{C}\right) \\
& =\frac{2 x \sqrt{3 t}+3 t-\left(2 y \sin t+\sin ^{2} t\right)}{C} .
\end{aligned}
$$

Since for every $x, y \in[0,1]$ it holds that

$$
2 y \sin t+\sin ^{2} t \leq 2 \sin t+\sin ^{2} t=\sin t(2+\sin t) \leq 3 t \leq 2 x \sqrt{3 t}+3 t,
$$

we have that $F(\mathbf{x}+\alpha(t)) \geq F(\mathbf{x})$, which means that $F$ is $\alpha$-increasing.

Example 3.6. Let $F:[0,1]^{2} \rightarrow[0,1]$ given by

$$
F(x, y)=\frac{1+3 x-y^{2}}{4} \text { for all } x, y \in[0,1]
$$

The function $F$ is $\alpha$-increasing for any curve $\alpha$ satisfying any of the following properties:

- $\alpha_{1}$ is increasing and $\alpha_{2}$ is decreasing; or

- $\alpha_{2}$ is increasing and $\alpha_{1} \geq \frac{2 \alpha_{2}+\alpha_{2}^{2}}{3}$; or

- the graph of $\alpha$ lies on the fourth quadrant of $\mathbb{R}^{2}$.

Example 3.7. Let $F:[0,1]^{2} \rightarrow[0,1]$ given by

$$
F(x, y)=\frac{x}{y+1} \quad \text { for all } x, y \in[0,1] .
$$

The function $F$ is $\alpha$-increasing for any curve $\alpha$ whose graph lies on the fourth quadrant of $\mathbb{R}^{2}$.

\subsection{Some considerations about the definition}

Moving along the image of the curve in $\mathbb{R}^{n}$, it is possible to go across the border of $[0,1]^{n}$ (as in the case of rays $\alpha(t)=t \mathbf{r}$ for $t \geq 0$ ). Hence the curve can leave the 
hypercube. A key question (directly related to Definition 3.1) is whether the curve re-enters the hypercube. This is the case in the plots of Figure 2 (where $n=2$ ).
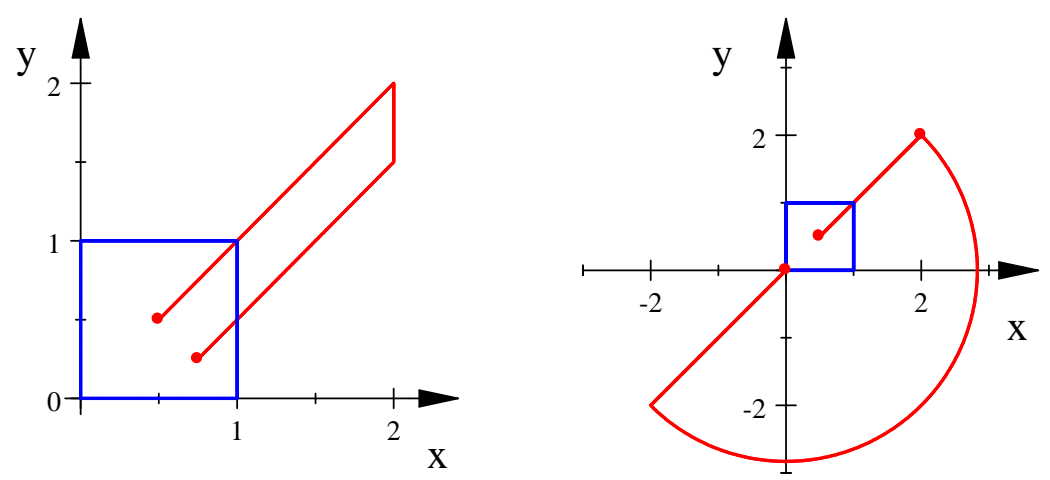

Figure 2. Two curves that leave and come back to the unit square $[0,1]^{2}$.

This question is critical in the following sense. Although we have introduced Definition 3.1, it would also be reasonable to study the class of fusion functions $F$ such that

$$
F(\mathbf{x}+\alpha(t)) \geq F(\mathbf{x}),
$$

for all $\mathbf{x} \in[0,1]^{n}$ and all $t \in I_{\alpha}$ such that $\mathbf{x}+\alpha(t) \in[0,1]^{n}$. However, the condition " $\mathbf{x}+\alpha(s) \in[0,1]^{n}$ for all $\left.\left.s \in\right] 0, t\right]$ " is important to guarantee that, when the path $s \mapsto \mathbf{x}+\alpha(s)$ leaves the hypercube $[0,1]^{n}$, we do not consider what happens beyond the first point in which the path goes across the border of $[0,1]^{n}$. If we do not impose such constraint and the curve leaves and reenters the hypercube, then an $\mathbf{r}$-increasing fusion function $F:[0,1]^{n} \rightarrow[0,1]$ would not necessarily be $\alpha$-increasing along curves whose first part coincides with the ray described by $\mathbf{r}$. In order to guarantee that $\mathbf{r}$ increasing fusion functions could also satisfy our definition for curves whose first part is a long enough ray, it could be interesting that, when $\mathbf{x}+\alpha(s) \notin[0,1]^{n}$, we can reduce the inequality, at most, to the interval $[0, s[$. Otherwise, the notion of increasingness along the curve might not be very intuitive.

Although condition (2) could seem more restrictive than (4), we avoid contexts like the ones given in Figure 2 or the following one (Figure 3).

Let $F:[0,1]^{2} \rightarrow[0,1]$ and $\alpha:[0,1] \rightarrow \mathbb{R}^{2}$ be given by $F(x, y)=\frac{1+x-y}{2}$, and

$$
\alpha(t)= \begin{cases}6 t(1,1), & \text { if } t \in\left[0, \frac{1}{3}\right], \\ 2 \sqrt{2}\left(\cos \left(-3 \pi t+\frac{5 \pi}{4}\right), \sin \left(-3 \pi t+\frac{5 \pi}{4}\right)\right), & \text { if } \left.t \in] \frac{1}{3}, \frac{2}{3}\right], \\ \left(\frac{15 t-18}{4}, \frac{27 t-26}{4}\right), & \text { if } \left.t \in] \frac{2}{3}, 1\right] .\end{cases}
$$

$F$ is clearly $(1,1)$-increasing. It is also $\alpha$-increasing according to Definition 3.1 , but would no longer be $\alpha$-increasing if condition (2) was replaced with (4). Indeed, if we take $\mathbf{x}_{0}=\left(\frac{3}{4}, \frac{3}{4}\right)$, the curve $t \mapsto \mathbf{x}_{0}+\alpha(t)$ leaves the unit square at $(1,1)$ but it enters again at the point $(0,1)$, and, in this case, we have no control on the values of $F\left(\mathbf{x}_{0}+\alpha(t)\right)$ : for example, $F\left(\mathbf{x}_{0}\right)=F\left(\frac{3}{4}, \frac{3}{4}\right)=0.5>0=F(0,1)=F\left(\mathbf{x}_{0}+\alpha(1)\right)$.

Remark 3.8. Since $\alpha(0)=\mathbf{0}$, inequality (2) is equivalent to imposing the following 


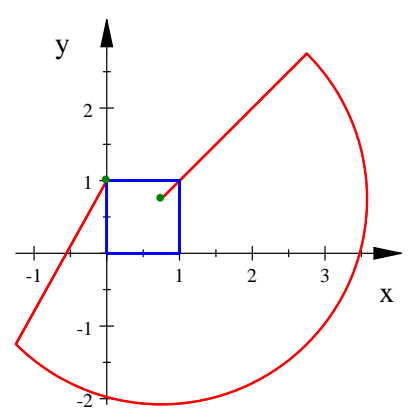

Figure 3. Graph of the curve $\alpha$ given in eq. (5).

condition:

for all $\mathbf{x} \in[0,1]^{n}$ and all $t \in I_{\alpha}$ such that $\mathbf{x}+\alpha(s) \in[0,1]^{n}$ for all $s \in[0, t]$, it follows that $\quad F(\mathbf{x}+\alpha(s)) \geq F(\mathbf{x})$ for all $s \in[0, t]$.

\section{3. $\quad \alpha$-monotonicity and monotonicity along the graph}

Although it may seem that Definition 3.1 implies monotonicity of the fusion function $F$ along the image of the curve $\alpha$, let us illustrate that this is not so.

Let $F:[0,1]^{n} \rightarrow[0,1]$ be a fusion function and let $\alpha: I_{\alpha} \rightarrow \mathbb{R}^{n}$ be a curve. Given an initial point $\mathbf{x} \in[0,1]^{n}$, let us consider

$$
J_{\mathbf{x}, \alpha}=\left\{t \in I_{\alpha}: \mathbf{x}+\alpha(t) \in[0,1]^{n}\right\} .
$$

Clearly $J_{\mathbf{x}, \alpha}$ is nonempty because $0 \in J_{\mathbf{x}, \alpha}$. On this set we can define the function $F_{\mathbf{x}, \alpha}: J_{\mathbf{x}, \alpha} \rightarrow[0,1]$ as follows:

$$
F_{\mathbf{x}, \alpha}(t)=F(\mathbf{x}+\alpha(t)) \quad \text { for all } t \in J_{\mathbf{x}, \alpha} .
$$

Although the subset $J_{\mathbf{x}, \alpha}$ is not necessarily an interval, when $J_{\mathbf{x}, \alpha}$ is an interval and $F$ is $\alpha$-increasing, the function $F_{\mathbf{x}, \alpha}$ is not necessarily increasing.

Taking $n=2$, let us consider the functions $F:[0,1]^{2} \rightarrow[0,1]$ and $\alpha:[0,1] \rightarrow \mathbb{R}^{2}$ defined as

$$
F(x, y)=\frac{1-x+y}{2}, \quad \alpha(t)= \begin{cases}(0,2 t), & \text { if } t \in\left[0, \frac{1}{2}\right], \\ (2 t-1,1), & \text { if } \left.t \in] \frac{1}{2}, 1\right] .\end{cases}
$$

It is inmediate that $F$ is $\alpha$-increasing. The image $\alpha\left(\left[0, \frac{1}{2}\right]\right)$ is the segment $\{0\} \times[0,1]$. When we go through $\alpha$ from $t=0$ to $t=\frac{1}{2}$, we start from a point $\mathbf{x}=(x, y)$ and we arrive at $\mathbf{x}+\alpha\left(\frac{1}{2}\right)=(x, y+1)$. The only way in which $\mathbf{x}+\alpha\left(\frac{1}{2}\right) \in[0,1]^{2}$ is starting from a point $\mathbf{x}_{0}=\left(x_{0}, 0\right)$, where $x_{0} \in[0,1]$. In this case,

$$
\mathbf{x}_{0}+\alpha(t) \in[0,1]^{2} \text { if and only if } t \in\left[0,1-\frac{x_{0}}{2}\right] .
$$


Therefore

$$
F_{\mathbf{x}_{0}, \alpha}(t)=F\left(\mathbf{x}_{0}+\alpha(t)\right)= \begin{cases}t+\frac{1-x_{0}}{2}, & \text { if } t \in\left[0, \frac{1}{2}\right], \\ \frac{3-x_{0}}{2}-t, & \text { if } \left.t \in] \frac{1}{2}, 1-\frac{x_{0}}{2}\right],\end{cases}
$$

whose graph is depicted in Figure 4.

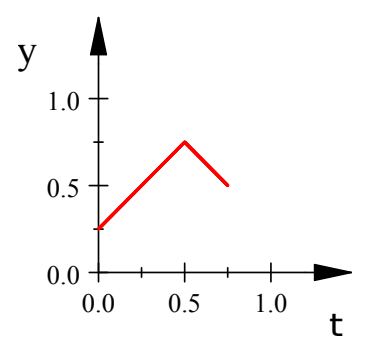

Figure 4. Plot of $t \mapsto F\left(\mathbf{x}_{0}+\alpha(t)\right)$ as in (6) starting from $\mathbf{x}_{0}=(0.25,0)$.

Hence $F$ is $\alpha$-increasing, since

$$
F_{\mathbf{x}_{0}, \alpha}(0)=F\left(\mathbf{x}_{0}\right)=\frac{1-x_{0}}{2} \leq F\left(\mathbf{x}_{0}+\alpha(t)\right)=F_{\mathbf{x}_{0}, \alpha}(t) \quad \text { for all } t \in\left[0,1-\frac{x_{0}}{2}\right] .
$$

However, the function $F_{\mathbf{x}_{0}, \alpha}:\left[0,1-\frac{x_{0}}{2}\right] \rightarrow \mathbb{R}$ is not increasing on the whole interval $\left[0,1-\frac{x_{0}}{2}\right]$ when $x_{0} \in[0,1[$ because

$$
F_{\mathbf{x}_{0}, \alpha}\left(\frac{1}{2}\right)=\frac{2-x_{0}}{2}>\frac{1}{2}=F_{\mathbf{x}_{0}, \alpha}\left(1-\frac{x_{0}}{2}\right) .
$$

Nevertheless, the converse holds.

Lemma 3.9. Given a fusion function $F:[0,1]^{n} \rightarrow[0,1]$ and a curve $\alpha: I_{\alpha} \rightarrow \mathbb{R}^{n}$, suppose that $F_{\mathbf{x}, \alpha}$ is increasing on $J_{\mathbf{x}, \alpha}$ whatever the point $\mathbf{x} \in[0,1]^{n}$. Then $F$ is $\alpha$-increasing.

Proof. Let $\mathbf{x} \in[0,1]^{n}$ and let $t \in I_{\alpha} \backslash\{0\}$ be such that $\mathbf{x}+\alpha(s) \in[0,1]^{n}$ for all $s \in] 0, t]$. Then $[0, t] \subseteq J_{\mathbf{x}, \alpha}$. As $F_{\mathbf{x}, \alpha}$ is increasing on $[0, t]$, then $F(\mathbf{x})=F_{\mathbf{x}, \alpha}(0) \leq$ $F_{\mathbf{x}, \alpha}(t)=F(\mathbf{x}+\alpha(t))$, so $F$ is $\alpha$-increasing.

\section{Properties of $\alpha$-monotonicity}

To start with, we analyze the relationship between $\alpha$-monotonicity and previous kinds of monotonicity. We start with the following remark that discusses the case for $n=1$.

Remark 4.1. Let $F:[0,1] \rightarrow[0,1]$ be a fusion function. On the one hand, if $\alpha$ is a continuous curve such that it is increasing and $\alpha \neq \alpha_{0}$, then $F$ is increasing (respectively, decreasing) if and only if $F$ is $\alpha$-increasing (respectively, $\alpha$-decreasing). Similarly, if $\alpha$ is decreasing, then $F$ is increasing (respectively, decreasing) if and only if $F$ is $\alpha$-decreasing (respectively, $\alpha$-increasing). 


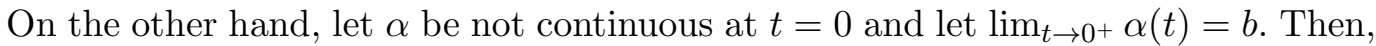
the following hold:

- If $b>1: \alpha$-increasingness is not a constraint for $F$, i.e., every $F$ is $\alpha$-increasing;

- if $b=1$ : If $F$ is $\alpha$-increasing, then $F(0) \leq F(1)$;

- if $0<b<1$ : If $F$ is $\alpha$-increasing, then $F(x) \leq F(y)$ for all $x<y$ such that $x+b \leq y$.

The following result goes in the direction of studying the relationship between $\alpha$ monotonicity and previous kinds of monotonicity.

Lemma 4.2. A fusion function $F:[0,1]^{n} \rightarrow[0,1]$ is increasing (respectively, decreasing) if and only if $F$ is $\alpha$-increasing (respectively, $\alpha$-decreasing) for every curve $\alpha: I_{\alpha} \rightarrow\left[0, \infty\left[^{n}\right.\right.$.

Proof. Assume first that $F$ is increasing (the decreasing case is analogous) and let $\alpha=\left(\alpha_{1}, \alpha_{2}, \ldots, \alpha_{n}\right): I_{\alpha} \rightarrow\left[0, \infty\left[{ }^{n}\right.\right.$ be a curve such that $\alpha_{i}(t) \geq 0$ for all $i \in\{1, \ldots, n\}$ and all $t \in I_{\alpha}$. Let $\mathbf{x} \in[0,1]^{n}$ and $t \in I_{\alpha} \backslash\{0\}$ be such that $\mathbf{x}+\alpha(s) \in[0,1]^{n}$ for all $s \in] 0, t]$. In particular,

$$
\left(x_{1}+\alpha_{1}(t), \ldots, x_{n}+\alpha_{n}(t)\right)=\mathbf{x}+\alpha(t) \in[0,1]^{n} .
$$

As $x_{i}+\alpha_{i}(t) \geq x_{i}$ for all $i \in\{1, \ldots, n\}$ and $F$ is increasing, then

$$
F(\mathbf{x}+\alpha(t))=F\left(x_{1}+\alpha_{1}(t), \ldots, x_{n}+\alpha_{n}(t)\right) \geq F\left(x_{1}, \ldots, x_{n}\right)=F(\mathbf{x}) .
$$

Conversely, suppose that $F$ is $\alpha$-increasing for every curve $\alpha: I_{\alpha} \rightarrow\left[0, \infty\left[{ }^{n}\right.\right.$. Let $\mathbf{x}, \mathbf{y} \in[0,1]^{n}$ be such that $x_{i} \leq y_{i}$ for all $i \in\{1, \ldots, n\}$ and consider the curve $\alpha:[0,1] \rightarrow \mathbb{R}^{n}$ defined by

$$
\alpha(t)=t(\mathbf{y}-\mathbf{x}) \quad \text { for all } t \in[0,1] .
$$

The curve $\alpha$ parametrizes the linear segment from $\alpha(0)=\mathbf{0}$ to $\alpha(1)=\mathbf{y}-\mathbf{x}$. Hence the curve $s \mapsto \mathbf{x}+\alpha(s)$ linearly goes from $\mathbf{x}$ to $\mathbf{y}$, which means that $\mathbf{x}+\alpha(s) \in[0,1]^{n}$ for all $s \in[0,1]$. Furthermore, since $0 \leq x_{i} \leq \alpha_{i}(t)=t\left(y_{i}-x_{i}\right) \leq t y_{i} \leq y_{i} \leq 1$ for all $i \in\{1, \ldots, n\}$ and all $t \in[0,1]$, our hypothesis implies that $F$ is $\alpha$-increasing. In particular, $F(\mathbf{y})=F(\mathbf{x}+\alpha(1)) \geq F(\mathbf{x})$, so $F$ is increasing.

A particular case of curves as in the previous result occurs when all components of $\alpha$ are equal, that is, if $\alpha_{i}(t)=\alpha_{j}(t) \geq 0$ for all $i, j \in\{1, \ldots, n\}$ and all $t \in I_{\alpha}$. In this case, we can characterize weak monotonicity.

Lemma 4.3. A fusion function $F:[0,1]^{n} \rightarrow[0,1]$ is weakly increasing if and only if $F$ is $\alpha$-increasing for every curve $\alpha: I_{\alpha} \rightarrow\left[0, \infty\left[{ }^{n}\right.\right.$ such that $\alpha_{i}(t)=\alpha_{j}(t)$ for all $i, j \in\{1, \ldots, n\}$ and all $t \in I_{\alpha}$.

Example 4.4. The mode function is $\alpha$-increasing for all curve $\alpha: I_{\alpha} \rightarrow\left[0, \infty\left[{ }^{n}\right.\right.$ such that $\alpha_{i}(t)=\alpha_{j}(t)$ for all $i, j \in\{1, \ldots, n\}$ and all $t \in I_{\alpha}$.

With respect to the relation of $\alpha$-monotonicity and directional monotonicity, if the curve $\alpha$ starts with a linear segment, then clearly this linear segment is related to a direction $\mathbf{r}$. Thus, for such a curve $\alpha$, if a fusion function $F$ is $\alpha$-monotone, then $F$ 
is also r-monotone. However, the converse implication does not hold, as shown in the following example.

Example 4.5. Let $F:[0,1]^{2} \rightarrow[0,1]$ given by

$$
F(x, y)= \begin{cases}0.5, & \text { if } \frac{x}{2}-\frac{1}{6}<y<\frac{x}{2}+\frac{2}{3}, \\ y, & \text { otherwise }\end{cases}
$$

and let $\alpha:[0,1] \rightarrow \mathbb{R}^{2}$ be the curve given by

$$
\alpha(t)=\left(t, \max \left(\frac{t}{2}, 2 t-1\right)\right) .
$$

On the one hand, it is not hard to show that the function $F$ is $\alpha$-increasing and also $(2,1)$-increasing.

On the other hand, however, the fusion function $G:[0,1]^{2} \rightarrow[0,1]$, given by

$$
G(x, y)=\frac{x-y+1}{2},
$$

is $(2,1)$-increasing but not $\alpha$-increasing, nor $\alpha$-decreasing. In fact, $G$ is $\mathbf{r}$-increasing for all non-zero $\mathbf{r}=\left(r_{1}, r_{2}\right)$ such that $r_{1} \geq r_{2}$, i.e., $G$ is cone-increasing with respect to the cone

$$
C=\left\{(x, y) \in \mathbb{R}^{2} \mid x \geq y\right\},
$$

which is a half-space. Therefore, $G$ is cone-increasing with respect to any subcone of $C$ but not $\alpha$-increasing, nor $\alpha$-decreasing.

Moreover, there also exist fusion functions that are $\alpha$-monotone, for certain curves $\alpha$, which are not directionally monotone for any direction $\mathbf{r}$. This is the case of the next example.

Example 4.6. Let $F:[0,1]^{2} \rightarrow[0,1]$ given by

$$
F(x, y)= \begin{cases}0, & \text { if } y=0 \text { or } y=1, \\ 2 x, & \text { if } x \leq 0.5 \text { and } 0<y<1 \\ -\frac{2}{3} x+\frac{4}{3}, & \text { otherwise }\end{cases}
$$

and let $\alpha:[0,1] \rightarrow \mathbb{R}^{2}$ be the curve given by

$$
\alpha(t)= \begin{cases}\left(\frac{2}{3}, 0\right), & \text { if } 0<t \leq \frac{2}{3} \\ (t, t), & \text { otherwise }\end{cases}
$$

Clearly, $F$ is not $\mathbf{r}$-monotone for any direction $\mathbf{r}=\left(r_{1}, r_{2}\right) \in \mathbb{R}^{2}$, since $F(0.5,0.5)=1$ and for any vector $\mathbf{r}$ there exists a constant $c>0$ for which $F\left(0.5+c r_{1}, 0.5+c r_{2}\right)<1$. This follows the fact that from the point $(0.5,0.5)$ the function strictly decreases in any direction $\left(r_{1}, r_{2}\right)$ such that $r_{1} \neq 0$. If $r_{1}=0$, it suffices to note that $F(0.5,0)=$ $F(0.5,1)=0$. Therefore, $F$ is not $\mathbf{r}$-increasing for any direction $\mathbf{r} \in \mathbb{R}^{2}$. Similarly, one can see that $F$ is not $\mathbf{r}$-decreasing for any direction $\mathbf{r} \in \mathbb{R}^{2}$. 
However, the fusion function $F$ is $\alpha$-increasing. Indeed, it suffices to check the points $\left(x \leq \frac{1}{3}\right.$ and $\left.0<y<1\right)$ because if $x>\frac{1}{3}$, then $(x, y)+\alpha(t) \notin[0,1]^{2}$ for $t>0$ and the cases in which $y \in\{0,1\}$ are trivial. Thus, let $(x, y) \in[0,1]^{2}$ such that $\left(x \leq \frac{1}{3}\right.$ and $\left.0<y<1\right)$. In this case, it holds that

$$
F(x, y)=2 x \leq-\frac{2}{3} x+\frac{8}{9}=F\left(x+\frac{2}{3}, y\right)=F((x, y)+\alpha(t)),
$$

if $0<t \leq \frac{2}{3}$. Similarly, $F(x, y) \leq F(x+t, y+t)=F((x, y)+\alpha(t))$ for $t>\frac{2}{3}$.

Hence, $F$ is an $\alpha$-increasing function that is not directionally monotone.

The definition of $\alpha$-monotonicity involves points of the hypercube of the form $\mathbf{x}+$ $\alpha(s)$. In a natural way, we can also consider points like $\mathbf{x}-\alpha(r)$. In the following result, we ensure that, under quite weak conditions, such points also belong to the hypercube.

Lemma 4.7. Let $\alpha: I_{\alpha} \rightarrow \mathbb{R}^{n}$ be a curve and assume that $\alpha$ is continuous at $t=0$. Then, for every $\mathbf{x} \in] 0,1\left[{ }^{n}\right.$, there exists $\delta>0$ such that the following condition holds for all $R, S \in[0, \delta]$ :

$$
\left(C_{R, S}^{\mathbf{x}, \alpha}\right) \quad\left\{\begin{array}{l}
\mathbf{x}-\alpha(R)+\alpha(r) \in[0,1]^{n} \text { for every } r \in[0, R], \quad \text { and } \\
\mathbf{x}+\alpha(s) \in[0,1]^{n} \text { for every } s \in[0, S] .
\end{array}\right.
$$

Proof. Let $\mathbf{x} \in] 0,1\left[^{n}\right.$. Since this is an open set, there exists $\eta>0$ such that the open ball $B(\mathbf{x}, 2 \eta) \subset] 0,1\left[{ }^{n}\right.$ (we consider open balls with respect to the Euclidean norm $\left.\|\cdot\|_{2}\right)$. Since $\alpha$ is continuous at $t=0$ and $\alpha(0)=\mathbf{0}$, there exists $\delta>0$ such that $\alpha(t) \in B(\mathbf{0}, \eta)$ for every $t \in[0, \delta]$. Let $R, S \in[0, \delta]$ be arbitrary. If $s \in[0, S] \subseteq[0, \delta]$, then

$$
\alpha(s) \in B(\mathbf{0}, \eta) \quad \Rightarrow \quad \mathbf{x}+\alpha(s) \in B(\mathbf{x}, \eta) \subset[0,1]^{n} .
$$

Similarly, let $r \in[0, R] \subseteq[0, \delta]$. Then if $\alpha(r) \in B(\mathbf{0}, \eta)$, then, in particular, $-\alpha(R) \in$ $B(\mathbf{0}, \eta)$ and therefore $\mathbf{x}-\alpha(R)+\alpha(r) \in B(\mathbf{x}, 2 \eta) \subset] 0,1\left[{ }^{n} \subset[0,1]^{n}\right.$. Hence condition $\left(C_{R, S}^{\mathbf{x}, \alpha}\right)$ holds.

The previous result guarantees that, under very weak hypotheses, condition $\left(C_{R, S}^{\mathbf{x}, \alpha}\right)$ holds for all $(R, S) \in[0, \delta] \times[0, \delta]$, where $\delta>0$.

Proposition 4.8. Given a curve $\alpha: I_{\alpha} \rightarrow \mathbb{R}^{n}$ and a point $\mathbf{x} \in[0,1]^{n}$, let $\Omega_{\mathbf{x}, \alpha}$ be the family of all pairs $\left(\theta_{1}, \theta_{2}\right) \in I_{\alpha} \times I_{\alpha}$ such that condition $\left(C_{R, S}^{\mathbf{x}, \alpha}\right)$ holds for all $R \in\left[0, \theta_{1}\right]$ and all $S \in\left[0, \theta_{2}\right]$. Then there are subintervals $I_{1}, I_{2} \subseteq I_{\alpha}$ such that $0 \in I_{1} \cap I_{2}$ and $\Omega_{\mathrm{x}, \alpha}=I_{1} \times I_{2}$.

Furthermore, if $\alpha$ is continuous, then $I_{1}$ and $I_{2}$ are closed subintervals of $I_{\alpha}$.

Proof. Let $I_{2}=\left\{S \in I_{\alpha}: \mathbf{x}+\alpha(s) \in[0,1]^{n}\right.$ for all $\left.s \in[0, S]\right\}$. Clearly $I_{2}$ is nonempty because $0 \in I_{2}$. Moreover, it is obvious that $I_{2}$ is an interval because if $S_{1}, S_{2} \in I_{2}$ and $S_{1} \leq S_{2}$, then $\left[S_{1}, S_{2}\right] \subseteq\left[0, S_{2}\right] \subseteq I_{2}$. In a similar way, we can deduce that

$$
I_{1}=\left\{R \in I_{\alpha}: \mathbf{x}-\alpha(R)+\alpha(r) \in[0,1]^{n} \text { for all } r \in[0, R]\right\}
$$


is also a subinterval of $I_{\alpha}$ containing 0 . Finally, $\Omega_{\mathbf{x}, \alpha}=I_{1} \times I_{2}$ because condition $\left(C_{R, S}^{\mathbf{x}, \alpha}\right)$ splits into two separate conditions.

Next, suppose that $\alpha$ is a continuous curve and we have to prove that $I_{1}$ and $I_{2}$ are closed subintervals of $I_{\alpha}$. We reason by using $I_{2}$. If $I_{2}=\{0\}$ or $I_{2}=I_{\alpha}$, then $I_{2}$ is closed on $I_{\alpha}$. In other case, suppose $I_{2} \neq\{0\}$ and $I_{2} \neq I_{\alpha}$. Hence, $I_{2}$ is bounded (it cannot be $\left[0, \infty[)\right.$. Let $\theta=\sup I_{2}$ and let $\left\{S_{m}\right\} \subseteq I_{2}$ be a strictly increasing sequence converging to $\theta$. Then $\theta \in I_{\alpha}$. As $\mathbf{x}+\alpha(s) \in[0,1]^{n}$ for all $s \in\left[0, S_{m}\right]$ and all $n \in \mathbb{N}$, then $\mathbf{x}+\alpha(s) \in[0,1]^{n}$ for all $s \in[0, \theta[$. In particular,

$$
0 \leq x_{i}+\alpha_{i}(s) \leq 1 \quad \text { for all } i \in\{1, \ldots, n\} \text { and all } s \in[0, \theta[.
$$

As $\left\{S_{m}\right\} \rightarrow \theta$ and $\alpha$ is continuous, then $\left\{\alpha\left(S_{m}\right)\right\} \rightarrow \alpha(\theta)$, which implies that $\left\{\alpha_{i}\left(S_{m}\right)\right\} \rightarrow \alpha_{i}(\theta)$ for all $i \in\{1, \ldots, n\}$. Letting $m \rightarrow \infty$ in (7), we deduce that

$$
0 \leq x_{i}+\alpha_{i}(\theta) \leq 1 \text { for all } i \in\{1, \ldots, n\},
$$

which guarantees that $\mathbf{x}+\alpha(\theta) \in[0,1]^{n}$. Hence $\theta \in I_{2}$ and we conclude that $I_{2}$ is a closed subinterval of $I_{\alpha}$.

Definition 3.1 can be extended in the following way.

Theorem 4.9. Given a fusion function $F:[0,1]^{n} \rightarrow[0,1]$ and a curve $\alpha: I_{\alpha} \rightarrow \mathbb{R}^{n}$, the following statements are equivalent.

(1) $F$ is $\alpha$-increasing.

(2) For all $\mathbf{x} \in[0,1]^{n}$ and all $r, s \in I_{\alpha}$ such that condition $\left(C_{r, s}^{\mathbf{x}, \alpha}\right)$ holds we have that

$$
F(\mathbf{x}-\alpha(r)) \leq F(\mathbf{x}) \leq F(\mathbf{x}+\alpha(s)) .
$$

(3) For all $\mathbf{x} \in[0,1]^{n}$ and all $s \in I_{\alpha}$ such that condition $\left(C_{0, s}^{\mathbf{x}, \alpha}\right)$ holds we have that

$$
F(\mathbf{x}) \leq F(\mathbf{x}+\alpha(s)) .
$$

Proof. Clearly $(2) \Rightarrow(3) \Rightarrow(1)$. Let us show that $(1) \Rightarrow(2)$. Let $r, s \in I_{\alpha}$ be such that $\left(C_{r, s}^{\mathbf{x}, \alpha}\right)$ holds. On the one hand, since $F$ is $\alpha$-increasing and $\mathbf{x}+\alpha(\tau) \in[0,1]^{n}$ for all $\tau \in[0, s]$, then $F(\mathbf{x}+\alpha(s)) \geq F(\mathbf{x})$. On the other hand, let $\mathbf{y}=\mathbf{x}-\alpha(r) \in \mathbb{R}^{n}$. As condition $\left(C_{r, s}^{\mathbf{x}, \alpha}\right)$ holds, then

$$
\mathbf{y}+\alpha(\tau)=\mathbf{x}-\alpha(r)+\alpha(\tau) \in[0,1]^{n} \quad \text { for all } \tau \in[0, r] .
$$

In particular, for $\tau=0$ we have that $\mathbf{y} \in[0,1]^{n}$. As $F$ is $\alpha$ increasing, $F(\mathbf{y}) \leq$ $F(\mathbf{y}+\alpha(r))$, which means that $F(\mathbf{x}-\alpha(r)) \leq F(\mathbf{x})$.

The last result has two main advantages: on the one hand, from a theoretical point of view, it completely characterizes the family of all fusion functions that are increasing along a curve $\alpha$; on the other hand, from a practical view-point, it upper and lower bounds the possible values of an $\alpha$-increasing fusion function with respect to its values on near points; hence, we could have a (weak) upper and lower control about the values of the fusion function. In this way, when we have proved that a fusion function is $\alpha$-increasing (maybe using the definition, which is easier to handle), we will have some information about the fusion function (for instance, if $F$ is continuous and $\alpha$ is 
continuous at $t=0$, then the values of $F$ along the image of the curve $t \mapsto F(\mathbf{x}+\alpha(t))$, $t \in I_{\alpha} \backslash\{0\}$, completely determines the value of $\left.F(\mathbf{x})\right)$. This boundedness will be the key property in order to study idempotency and averaging behaviour of $\alpha$-monotone fusion functions even if they are not continuous (see Section 6).

\section{5. $\alpha$-monotonicity from an analytical and an algebraic point of view}

\subsection{Discussion on the domain of curves}

In our study of monotonicity along a curve, we are considering curves $\alpha: I_{\alpha} \rightarrow \mathbb{R}^{n}$ such that $\alpha(0)=\mathbf{0}$, where the domain $I_{\alpha}$ can be of three different types: $[0, \infty[,[0, \theta]$ or $\left[0, \theta[\right.$ (for some $\theta \in] 0, \infty\left[\right.$ ). Until now, the domain $I_{\alpha}$ has not played a significant role. In fact, it is easy to replace the domain by another one of the same kind, as in the following result.

Proposition 5.1. Given a curve $\alpha: I_{\alpha} \rightarrow \mathbb{R}^{n}$, let $J$ be an interval of the same class as $I_{\alpha}$, let $f: J \rightarrow I_{\alpha}$ be a strictly increasing bijection and consider the curve $\alpha \circ f: J \rightarrow \mathbb{R}^{n}$. Then any fusion function $F$ is $\alpha$-increasing (respectively, $\alpha$-decreasing) if and only if $F$ is $(\alpha \circ f)$-increasing (respectively, $(\alpha \circ f)$-decreasing).

Proof. Suppose, for instance, that $I_{\alpha}=\left[0, \theta_{1}\left[\right.\right.$ and $J=\left[0, \theta_{2}\left[\right.\right.$, where $\left.\theta_{1}, \theta_{2} \in\right] 0, \infty[$ (or even $\theta_{1}=\theta_{2}=\infty$ ). As $f$ is an strictly increasing bijection, then necessarily $f(0)=0$, and each subinterval $\left[0, t^{\prime}\right] \subseteq J$ is transformed, by $f$, on $\left[0, f\left(t^{\prime}\right)\right] \subseteq I_{\alpha}$. Suppose that $F$ is $\alpha$-increasing. In order to prove that $F$ is $(\alpha \circ f)$-increasing, let $\mathbf{x} \in[0,1]^{n}$ and let $t^{\prime} \in J \backslash\{0\}$ be such that $\mathbf{x}+(\alpha \circ f)\left(s^{\prime}\right) \in[0,1]^{n}$ for all $\left.\left.s^{\prime} \in\right] 0, t^{\prime}\right]$. Let $t=f\left(t^{\prime}\right) \in I_{\alpha} \backslash\{0\}$. As $f$ is a bijection, $f\left(\left[0, t^{\prime}\right]\right)=[0, t]$. Furthermore, for all $s \in[0, t]$, the point $\left.\left.s^{\prime}=f^{-1}(s) \in\right] 0, t^{\prime}\right]$ and

$$
\mathbf{x}+\alpha(s)=\mathbf{x}+\alpha\left(f\left(s^{\prime}\right)\right)=\mathbf{x}+(\alpha \circ f)\left(s^{\prime}\right) \in[0,1]^{n} .
$$

As $F$ is $\alpha$-increasing, then $F(\mathbf{x}+\alpha(t)) \geq F(\mathbf{x})$, so

$$
F\left(\mathbf{x}+(\alpha \circ f)\left(t^{\prime}\right)\right)=F\left(\mathbf{x}+\alpha\left(f\left(t^{\prime}\right)\right)\right)=F(\mathbf{x}+\alpha(t)) \geq F(\mathbf{x}),
$$

which means that $F$ is $(\alpha \circ f)$-increasing. The converse is similar.

From a geometrical point of view, the previous result means that the interval $I_{\alpha}$ is not essential in order to study monotonicity along a curve: the only important thing is the kind of the involved interval. As a consequence, we can reduce our study to curves defined on $[0,1[$ or $[0,1]$.

Corollary 5.2. Given a curve $\alpha: I_{\alpha} \rightarrow \mathbb{R}^{n}$, let

$$
I_{\alpha}^{\prime}=\left\{\begin{array}{l}
{\left[0,1\left[, \quad \text { if } I_{\alpha} \text { is unbounded or (bounded and open on }[0, \infty[\text { ), }\right.\right.} \\
{[0,1], \quad \text { if } I_{\alpha} \text { is bounded and closed on }[0, \infty[.}
\end{array}\right.
$$

Then there is a strictly increasing bijection $f: I_{\alpha}^{\prime} \rightarrow I_{\alpha}$. Furthermore, any fusion function $F$ is $\alpha$-increasing (respectively, $\alpha$-decreasing) if and only if $F$ is $(\alpha \circ f)$ increasing (respectively, $(\alpha \circ f)$-decreasing). 
Proof. We have only to consider the bijections $f_{1}:\left[0,1\left[\rightarrow\left[0, \theta\left[, f_{2}:[0,1] \rightarrow[0, \theta]\right.\right.\right.\right.$ and $f_{3}:[0,1[\rightarrow[0, \infty[$ given by

$$
f_{1}(t)=f_{2}(t)=\theta t \quad \text { and } \quad f_{3}(t)=\frac{t}{1-t}
$$

for all $t$ on their corresponding domains (where $\theta \in] 0, \infty[$ ).

The advantage of the curve $\alpha \circ f: I_{\alpha}^{\prime} \rightarrow \mathbb{R}^{n}$ is that its domain is either $[0,1[$ or $[0,1]$. Hence we can reduce our study to curves of the form $\alpha:\left[0,1\left[\rightarrow \mathbb{R}^{n}\right.\right.$ or $\alpha$ : $[0,1] \rightarrow \mathbb{R}^{n}$. However, both of them are distinct in nature. Every curve $\alpha:\left[0,1\left[\rightarrow \mathbb{R}^{n}\right.\right.$ can be extended to a curve $\bar{\alpha}:[0,1] \rightarrow \mathbb{R}^{n}$ by defining $\bar{\alpha}(1)$ arbitrarily in $\mathbb{R}^{n}$. If $\alpha$ is continuous and $\lim _{t \rightarrow 1^{-}} \alpha(t)$ exists, then we can consider a unique continuous extension $\bar{\alpha}:[0,1] \rightarrow \mathbb{R}^{n}$. This is not the case if such limit does not exist, as in the following example.

$$
\alpha:\left[0,1\left[\rightarrow \mathbb{R}^{2}, \quad \alpha(t)=\left(t, \frac{t}{1-t}\right) \quad \text { for all } t \in[0,1[.\right.\right.
$$

The previous curve cannot be extended to $t=1$ in a continuous way. As a consequence, in many cases, we will need to develop distinct techniques for curves defined on $[0,1[$ or $[0,1]$. This is the case of the algebraic construction in the following subsection.

\section{2. $\alpha$-monotonicity from an algebraic point of view}

In this subsection, we study monotonicity along curves defined on $[0,1]$. In general, given two curves $\alpha, \beta:[0,1] \rightarrow \mathbb{R}^{n}$, we can consider the sum $\alpha+\beta:[0,1] \rightarrow \mathbb{R}^{n}$ given by $(\alpha+\beta)(t)=\alpha(t)+\beta(t)$ for all $t \in[0,1]$. This sum also satisfies $(\alpha+\beta)(0)=\mathbf{0}$. However, a fusion function which is $\alpha$-increasing and $\beta$-increasing is not necessarily $(\alpha+\beta)$-increasing, as the following example proves.

Example 5.3. Let $F:[0,1]^{2} \rightarrow[0,1]$ be the fusion function given by

$$
F(x, y)= \begin{cases}0, & \text { if } x=y=1 \\ 1, & \text { otherwise }\end{cases}
$$

If we take the vectors $\mathbf{r}_{1}=(2,-1)$ and $\mathbf{r}_{2}=(-1,2)$, it is easy to show that $F$ is $\mathbf{r}_{1}$-increasing and $\mathbf{r}_{2}$-increasing. Hence, it is $\alpha$-increasing and $\beta$-increasing, where $\alpha$ and $\beta$ are the rays $\alpha(t)=t \mathbf{r}_{1}$ and $\beta(t)=t \mathbf{r}_{2}$ for all $t \in[0,1]$. However, $F$ is not $(\alpha+\beta)$-increasing because $(\alpha+\beta)(t)=t\left(\mathbf{r}_{1}+\mathbf{r}_{2}\right)=(t, t)$ is the ray in the direction of the vector $(1,1)$.

In this subsection we introduce a new operation $\star$ such that if $F$ is $\alpha$-increasing and $\beta$-increasing, then it is also $(\alpha \star \beta)$-increasing.

Definition 5.4. Let $\alpha, \beta:[0,1] \rightarrow \mathbb{R}^{n}$ be two curves. We define a new curve $\alpha \star \beta$ : 
$[0,1] \rightarrow \mathbb{R}^{n}$ as

$$
(\alpha \star \beta)(t)= \begin{cases}\alpha(2 t), & \text { if } t \in\left[0, \frac{1}{2}\right], \\ \alpha(1)+\beta(2 t-1), & \text { if } \left.t \in] \frac{1}{2}, 1\right] .\end{cases}
$$

Note that $\alpha \star \beta$ is the juxtaposition curve of $\alpha$ and $\beta$. Moreover, at $t=\frac{1}{2}$ the function equals $\alpha(1)$.

Given a fusion function $F$, let

$$
\begin{aligned}
& \operatorname{Mon}^{\uparrow}(F)=\left\{\alpha:[0,1] \rightarrow \mathbb{R}^{n}: F \text { is } \alpha \text {-increasing }\right\} \\
& \operatorname{Mon}^{\downarrow}(F)=\left\{\alpha:[0,1] \rightarrow \mathbb{R}^{n}: F \text { is } \alpha \text {-decreasing }\right\} .
\end{aligned} \text { and }
$$

Clearly $\operatorname{Mon}^{\uparrow}(F)$ and $\operatorname{Mon}^{\downarrow}(F)$ are nonempty since, for instance, $\alpha_{\mathbf{0}} \in \operatorname{Mon}^{\uparrow}(F) \cap$ $\operatorname{Mon}^{\downarrow}(F)$. The following result directly follows from Proposition 5.1.

Corollary 5.5. If $F$ is a fusion function and $f:[0,1] \rightarrow[0,1]$ is a strictly increasing bijection, then $\alpha \in \operatorname{Mon}^{\uparrow}(F)$ (respectively, $\alpha \in \operatorname{Mon}^{\downarrow}(F)$ ) if and only if $\alpha \circ f \in$ $\operatorname{Mon}^{\uparrow}(F)$ (respectively, $\alpha \circ f \in \operatorname{Mon}^{\downarrow}(F)$ ).

In the following lemma, we show that $\operatorname{Mon}^{\uparrow}(F)$ is closed for the operation $\star$ (the reader can translate the following results to $\operatorname{Mon}^{\downarrow}(F)$ ).

Lemma 5.6. If $F:[0,1]^{n} \rightarrow[0,1]$ is a fusion function and $\alpha, \beta \in \operatorname{Mon}^{\uparrow}(F)$, then $\alpha \star \beta \in \operatorname{Mon}^{\uparrow}(F)$. That is, $\star$ is a binary operation on $\operatorname{Mon}^{\uparrow}(F)$ and

$$
F(\mathbf{x}+(\alpha \star \beta)(t)) \geq F(\mathbf{x})
$$

for all $\mathbf{x} \in[0,1]^{n}$ and all $t \in[0,1]$ such that $\mathbf{x}+(\alpha \star \beta)(s) \in[0,1]^{n}$ for all $s \in[0, t]$.

Proof. Let $\mathbf{x} \in[0,1]^{n}$ and let $\left.\left.t \in\right] 0,1\right]$ be such that $\left.\left.\mathbf{x}+(\alpha \star \beta)(s) \in\right] 0,1\right]^{n}$ for all $s \in[0, t]$. We have to prove that $F(\mathbf{x}+(\alpha \star \beta)(t)) \geq F(\mathbf{x})$. We consider two cases.

Case 1: Suppose that $t \in\left[0, \frac{1}{2}\right]$. Let $t^{\prime}=2 t \in[0,1]$. Then, for all $s \in\left[0, t^{\prime}\right]$ we have that $\frac{s}{2} \in[0, t] \subseteq\left[0, \frac{1}{2}\right]$ and

$$
\mathbf{x}+\alpha(s)=\mathbf{x}+\alpha\left(2 \frac{s}{2}\right)=\mathbf{x}+(\alpha \star \beta)\left(\frac{s}{2}\right) \in[0,1]^{n} .
$$

Since $F$ is $\alpha$-increasing, we deduce that

$$
F(\mathbf{x}+(\alpha \star \beta)(t))=F(\mathbf{x}+\alpha(2 t))=F\left(\mathbf{x}+\alpha\left(t^{\prime}\right)\right) \geq F(\mathbf{x}) .
$$

Case 2: Suppose that $\left.t \in] \frac{1}{2}, 1\right]$. In this case, let $\left.\left.t^{\prime}=2 t-1 \in\right] 0,1\right]$ and $\mathbf{y}=\mathbf{x}+\alpha(1)=$ $\mathbf{x}+(\alpha \star \beta)\left(\frac{1}{2}\right) \in[0,1]^{n}$. Let $s \in\left[0, t^{\prime}\right]$ be arbitrary. Then $\frac{s+1}{2} \in[0, t]$ and

$$
\mathbf{y}+\beta(s)=(\mathbf{x}+\alpha(1))+\beta\left(2 \frac{s+1}{2}-1\right)=\mathbf{x}+(\alpha \star \beta)\left(\frac{s+1}{2}\right) \in[0,1]^{n} .
$$

Since $F$ is $\beta$-increasing,

$$
F(\mathbf{x}+(\alpha \star \beta)(t))=F(\mathbf{x}+\alpha(1)+\beta(2 t-1))=F(\mathbf{y}+\beta(2 t-1))=F\left(\mathbf{y}+\beta\left(t^{\prime}\right)\right) \geq F(\mathbf{x}) .
$$


In any case, we deduce that $\alpha \star \beta \in \operatorname{Mon}^{\uparrow}(F)$.

Remark 5.7. Definition 5.4 and Lemma 5.6 cannot be considered, in the same way and with the same geometric interpretation, for curves of the form $\alpha:\left[0,1\left[\rightarrow \mathbb{R}^{n}\right.\right.$. In order to define $(\alpha \star \beta)\left(\frac{1}{2}\right)$, it would be necessary, for instance, to impose that $\alpha$ is continuous and that $\lim _{t \rightarrow 1} \alpha(t)$ exists.

Definition 5.8. Let $\Phi$ denote the family of all continuous and strictly increasing functions $\phi:[0,1] \rightarrow[0,1]$ such that $\phi^{-1}(\{0\})=\{0\}$ and $\phi^{-1}(\{1\})=\{1\}$.

In the next definition, we introduce a binary relation on the set $\operatorname{Mon}^{\uparrow}(F)$.

Definition 5.9. Given a fusion function $F:[0,1]^{n} \rightarrow[0,1]$ and $\alpha, \beta \in \operatorname{Mon}^{\uparrow}(F)$, we will write $\alpha \sim \beta$ if there exist $\phi, \psi \in \Phi$ such that

$$
\alpha \circ \phi=\beta \quad \text { and } \quad \beta \circ \psi=\alpha .
$$

In other words, the curve $\alpha$ is a reparametrization of the curve $\beta$, and viceversa.

Proposition 5.10. If $F$ is a fusion function and $\alpha, \beta \in \operatorname{Mon}^{\uparrow}(F)$ are such that $\alpha \sim \beta$, then both curves $\alpha, \beta:[0,1] \rightarrow \mathbb{R}^{n}$ have the same image (that is, $\alpha([0,1])=\beta([0,1])$ ).

The following result describes the most important properties of the binary relation $\sim$.

Theorem 5.11. Given a fusion function $F$ and $\alpha, \beta, \gamma \in \operatorname{Mon}^{\uparrow}(F)$, the following properties hold.

(1) Reflexivity: $\alpha \sim \alpha$.

(2) Symmetry: if $\alpha \sim \beta$, then $\beta \sim \alpha$.

(3) Transitivity: if $\alpha \sim \beta$ and $\beta \sim \gamma$, then $\alpha \sim \gamma$.

As a consequence, $\sim$ is an equivalence relation on $\operatorname{Mon}^{\uparrow}(F)$.

Proof. Items (1) and (2) are trivial.

(3) Suppose that $\alpha \sim \beta$ and $\beta \sim \gamma$. Then there exist $\phi_{1}, \psi_{1}, \phi_{2}, \psi_{2} \in \Phi$ such that

$$
\alpha \circ \phi_{1}=\beta, \quad \beta \circ \psi_{1}=\alpha, \quad \beta \circ \phi_{2}=\gamma \quad \text { and } \quad \gamma \circ \psi_{2}=\beta \text {. }
$$

Let $\phi=\phi_{1} \circ \phi_{2} \in \Phi$ and $\psi=\psi_{2} \circ \psi_{1} \in \Phi$. Therefore

$$
\begin{aligned}
& \alpha \circ \phi=\alpha \circ\left(\phi_{1} \circ \phi_{2}\right)=\left(\alpha \circ \phi_{1}\right) \circ \phi_{2}=\beta \circ \phi_{2}=\gamma \text { and } \\
& \gamma \circ \psi=\gamma \circ\left(\psi_{2} \circ \psi_{1}\right)=\left(\gamma \circ \psi_{2}\right) \circ \psi_{1}=\beta \circ \psi_{1}=\alpha,
\end{aligned}
$$

so $\alpha \sim \gamma$.

The following result describes some relationships between the binary relation $\sim$ and the operation $\star$.

Theorem 5.12. Given a fusion function $F$, the following properties hold.

(1) If $\alpha_{1}, \alpha_{2}, \beta_{1}, \beta_{2} \in \operatorname{Mon}^{\uparrow}(F)$ are such that $\alpha_{1} \sim \alpha_{2}$ and $\beta_{1} \sim \beta_{2}$, then $\alpha_{1} \star \beta_{1} \sim$ $\alpha_{2} \star \beta_{2}$.

(2) Associativity: $(\alpha \star \beta) \star \gamma \sim \alpha \star(\beta \star \gamma)$ for all $\alpha, \beta, \gamma \in \operatorname{Mon}^{\uparrow}(F)$. 
Proof. (1) Suppose that there exist $\phi_{1}, \psi_{1}, \phi_{2}, \psi_{2} \in \Phi$ such that

$$
\alpha_{1} \circ \phi_{1}=\alpha_{2}, \quad \alpha_{2} \circ \psi_{1}=\alpha_{1}, \quad \beta_{1} \circ \phi_{2}=\beta_{2} \quad \text { and } \quad \beta_{2} \circ \psi_{2}=\beta_{1} \text {. }
$$

Let $\phi:[0,1] \rightarrow[0,1]$ be as follows:

$$
\phi(t)= \begin{cases}\frac{\phi_{1}(2 t)}{2}, & \text { if } t \in\left[0, \frac{1}{2}\right], \\ \frac{1+\phi_{2}(2 t-1)}{2}, & \text { if } \left.t \in] \frac{1}{2}, 1\right] .\end{cases}
$$

Clearly $\phi(0)=\frac{\phi_{1}(0)}{2}=0$ and $\phi(1)=\frac{1+\phi_{2}(1)}{2}=1$. Note that

$$
\begin{aligned}
& \phi(t)=0 \Rightarrow \frac{\phi_{1}(2 t)}{2}=0 \Rightarrow \phi_{1}(2 t)=0 \Rightarrow 2 t=0 \Rightarrow t=0, \\
& \phi(t)=1 \Rightarrow \frac{1+\phi_{2}(2 t-1)}{2}=1 \Rightarrow \phi_{2}(2 t-1)=1 \Rightarrow 2 t-1=1 \Rightarrow t=1 .
\end{aligned}
$$

Then $\phi^{-1}(\{0\})=\{0\}$ and $\phi^{-1}(\{1\})=\{1\}$. Since $\phi_{1}$ and $\phi_{2}$ are continuous, then

$$
\begin{aligned}
& \lim _{t \rightarrow 1 / 2^{-}} \phi(t)=\lim _{t \rightarrow 1 / 2^{-}} \frac{\phi_{1}(2 t)}{2}=\frac{\phi_{1}(1)}{2}=\frac{1}{2}=\phi\left(\frac{1}{2}\right) \quad \text { and } \\
& \lim _{t \rightarrow 1 / 2^{+}} \phi(t)=\lim _{t \rightarrow 1 / 2^{+}} \frac{1+\phi_{2}(2 t-1)}{2}=\frac{1+\phi_{2}(0)}{2}=\frac{1}{2}
\end{aligned}
$$

so $\phi$ is continuous on $[0,1]$. Furthermore, $\phi(t) \leq \frac{1}{2} \leq \phi(s)$ for all $t \in\left[0, \frac{1}{2}\right]$ and all $s \in\left[\frac{1}{2}, 1\right]$. As $\phi_{1}$ and $\phi_{2}$ are increasing, then $\phi$ is also increasing. Therefore, $\phi \in \Phi$. In addition to this, we claim that

$$
\phi(t)=\frac{1}{2} \quad \text { if and only if } \quad t=\frac{1}{2} .
$$

Clearly $\phi\left(\frac{1}{2}\right)=\frac{\phi_{1}(1)}{2}=\frac{1}{2}$. On the other hand,

$$
\begin{aligned}
& \frac{\phi_{1}(2 t)}{2}=\frac{1}{2} \Rightarrow \phi_{1}(2 t)=1 \Rightarrow 2 t=1 \Rightarrow t=\frac{1}{2} ; \\
& \frac{1+\phi_{2}(2 t-1)}{2}=\frac{1}{2} \Rightarrow \phi_{2}(2 t-1)=0 \Rightarrow 2 t-1=0 \Rightarrow t=\frac{1}{2}
\end{aligned}
$$

(the second case is impossible because we suppose that $\left.t \in] \frac{1}{2}, 1\right]$ ). As a consequence

$$
\begin{array}{ll}
\phi(t) \in\left[0, \frac{1}{2}\right] \quad \Leftrightarrow t \in\left[0, \frac{1}{2}\right] \quad \text { and } \\
\phi(t) \in\left[\frac{1}{2}, 1\right] \quad \Leftrightarrow t \in\left[\frac{1}{2}, 1\right] .
\end{array}
$$


Hence, for all $t \in[0,1]$,

$$
\begin{aligned}
\left(\left(\alpha_{1} \star \beta_{1}\right) \circ \phi\right)(t) & =\left(\alpha_{1} \star \beta_{1}\right)(\phi(t))= \begin{cases}\alpha_{1}(2 \phi(t)), & \text { if } \phi(t) \in\left[0, \frac{1}{2}\right], \\
\alpha_{1}(1)+\beta_{1}(2 \phi(t)-1), & \text { if } \left.\phi(t) \in] \frac{1}{2}, 1\right]\end{cases} \\
& = \begin{cases}\alpha_{1}\left(2 \frac{\phi_{1}(2 t)}{2}\right), & \text { if } t \in\left[0, \frac{1}{2}\right], \\
\alpha_{1}(1)+\beta_{1}\left(2 \frac{1+\phi_{2}(2 t-1)}{2}-1\right), & \text { if } \left.t \in] \frac{1}{2}, 1\right]\end{cases} \\
& = \begin{cases}\alpha_{1}\left(\phi_{1}(2 t)\right), & \text { if } t \in\left[0, \frac{1}{2}\right], \\
\alpha_{2}(1)+\beta_{1}\left(\phi_{2}(2 t-1)\right), & \text { if } \left.t \in] \frac{1}{2}, 1\right]\end{cases} \\
& = \begin{cases}\left(\alpha_{1} \circ \phi_{1}\right)(2 t), & \text { if } t \in\left[0, \frac{1}{2}\right], \\
\alpha_{2}(1)+\left(\beta_{1} \circ \phi_{2}\right)(2 t-1), & \text { if } \left.t \in] \frac{1}{2}, 1\right]\end{cases} \\
& = \begin{cases}\alpha_{2}(2 t), & \text { if } t \in\left[0, \frac{1}{2}\right], \\
\alpha_{2}(1)+\beta_{2}(2 t-1), & \text { if } \left.t \in] \frac{1}{2}, 1\right]\end{cases} \\
& =\left(\begin{array}{l}
\left.\alpha_{2} \star \beta_{2}\right)(t) .
\end{array}\right.
\end{aligned}
$$

This proves that $\left(\alpha_{1} \star \beta_{1}\right) \circ \phi=\alpha_{2} \star \beta_{2}$. In a similar way we can consider a function $\psi \in \Phi$ such that $\left(\alpha_{2} \star \beta_{2}\right) \circ \psi=\alpha_{1} \star \beta_{1}$, which concludes that $\alpha_{1} \star \beta_{1} \sim \alpha_{2} \star \beta_{2}$.

(2) Let us consider the function $\phi:[0,1] \rightarrow[0,1]$ defined as follows (see Figure 5):

$$
\phi(t)= \begin{cases}\frac{t}{2}, & \text { if } t \in\left[0, \frac{1}{2}\right], \\ t-\frac{1}{4}, & \text { if } t \in\left[\frac{1}{2}, \frac{3}{4}\right] \\ 2 t-1, & \text { if }\left[\frac{3}{4}, 1\right] .\end{cases}
$$

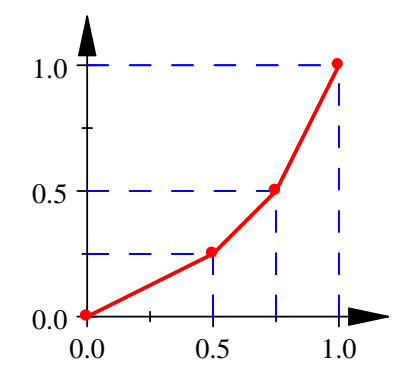

Figure 5. Plot of function $\phi$ given by eq. (8). 
Clearly $\phi \in \Phi$. Note that

$$
\begin{aligned}
& \phi(t) \in\left[0, \frac{1}{4}\right] \quad \Leftrightarrow t \in\left[0, \frac{1}{2}\right], \\
&\left.\left.\left.\phi(t) \in] \frac{1}{4}, \frac{1}{2}\right] \quad \Leftrightarrow \quad t \in\right] \frac{1}{2}, \frac{3}{4}\right] \quad \text { and } \\
&\left.\left.\left.\phi(t) \in] \frac{1}{2}, 1\right] \quad \Leftrightarrow \quad t \in\right] \frac{3}{4}, 1\right] .
\end{aligned}
$$

On the one hand, for all $t \in[0,1]$,

$$
\begin{aligned}
& (\alpha \star(\beta \star \gamma))(t)= \begin{cases}\alpha(2 t), & \text { if } t \in\left[0, \frac{1}{2}\right], \\
\alpha(1)+(\beta \star \gamma)(2 t-1), & \text { if } \left.t \in] \frac{1}{2}, 1\right]\end{cases} \\
& = \begin{cases}\alpha(2 t), & \text { if } t \in\left[0, \frac{1}{2}\right], \\
\alpha(1)+\beta(2(2 t-1)) & \text { if } \left.t \in] \frac{1}{2}, \frac{3}{4}\right], \\
\alpha(1)+\beta(1)+\gamma(2(2 t-1)-1), & \text { if } \left.t \in] \frac{1}{2}, 1\right]\end{cases} \\
& = \begin{cases}\alpha(2 t), & \text { if } t \in\left[0, \frac{1}{2}\right], \\
\alpha(1)+\beta(4 t-2) & \text { if } \left.t \in] \frac{1}{2}, \frac{3}{4}\right], \\
\alpha(1)+\beta(1)+\gamma(4 t-3), & \text { if } \left.t \in] \frac{1}{2}, 1\right] .\end{cases}
\end{aligned}
$$


On the other hand,

$$
\begin{aligned}
& (((\alpha \star \beta) \star \gamma) \circ \phi)(t)=((\alpha \star \beta) \star \gamma)(\phi(t)) \\
& = \begin{cases}(\alpha \star \beta)(2 \phi(t)), & \text { if } \phi(t) \in\left[0, \frac{1}{2}\right], \\
(\alpha \star \beta)(1)+\gamma(2 \phi(t)-1), & \text { if } \left.\phi(t) \in] \frac{1}{2}, 1\right]\end{cases} \\
& = \begin{cases}\alpha(2 * 2 \phi(t)), & \text { if } \phi(t) \in\left[0, \frac{1}{4}\right], \\
\alpha(1)+\beta(2 * 2 \phi(t)-1), & \text { if } \left.\phi(t) \in] \frac{1}{4}, \frac{1}{2}\right], \\
\alpha(1)+\beta(1)+\gamma(2 \phi(t)-1), & \text { if } \left.\phi(t) \in] \frac{1}{2}, 1\right]\end{cases} \\
& = \begin{cases}\alpha(4 \phi(t)), & \text { if } t \in\left[0, \frac{1}{2}\right], \\
\alpha(1)+\beta(4 \phi(t)-1), & \text { if } \left.t \in] \frac{1}{2}, \frac{3}{4}\right], \\
\alpha(1)+\beta(1)+\gamma(2 \phi(t)-1), & \text { if } \left.t \in] \frac{3}{4}, 1\right]\end{cases} \\
& = \begin{cases}\alpha\left(4 \frac{t}{2}\right), & \text { if } t \in\left[0, \frac{1}{2}\right], \\
\alpha(1)+\beta\left(4\left(t-\frac{1}{4}\right)-1\right), & \text { if } \left.t \in] \frac{1}{2}, \frac{3}{4}\right], \\
\alpha(1)+\beta(1)+\gamma(2(2 t-1)-1), & \text { if } \left.t \in] \frac{3}{4}, 1\right]\end{cases} \\
& = \begin{cases}\alpha(2 t), & \text { if } t \in\left[0, \frac{1}{2}\right], \\
\alpha(1)+\beta(4 t-2), & \text { if } \left.t \in] \frac{1}{2}, \frac{3}{4}\right], \\
\alpha(1)+\beta(1)+\gamma(4 t-3), & \text { if } \left.t \in] \frac{3}{4}, 1\right]\end{cases} \\
& =(\alpha \star(\beta \star \gamma))(t) \text {. }
\end{aligned}
$$

This proves that $((\alpha \star \beta) \star \gamma) \circ \phi=\alpha \star(\beta \star \gamma)$. In a similar way we can consider a function $\psi \in \Phi$ such that $(\alpha \star(\beta \star \gamma)) \circ \psi=(\alpha \star \beta) \star \gamma$. Hence, $(\alpha \star \beta) \star \gamma \sim$ $\alpha \star(\beta \star \gamma)$.

Remark 5.13. Although it may seem counterintuitive, it holds that $\alpha \star \alpha_{\mathbf{0}} \nsim \alpha$ and $\alpha_{0} \star \alpha \not \alpha$ for $\alpha \in \operatorname{Mon}^{\uparrow}(F)$.

As a consequence of Theorem 5.12, the operation $\star$ is well-defined on the quotient space and it is associative.

Corollary 5.14. Given a fusion function $F$, let $\frac{\operatorname{Mon}^{\uparrow}(F)}{\sim}$ be the quotient space of $\operatorname{Mon}^{\uparrow}(F)$ over the equivalence relation $\sim$ and let us define

$$
\widetilde{\star}: \frac{\operatorname{Mon}^{\uparrow}(F)}{\sim} \times \frac{\operatorname{Mon}^{\uparrow}(F)}{\sim} \rightarrow \frac{\operatorname{Mon}^{\uparrow}(F)}{\sim}, \quad([\alpha],[\beta]) \mapsto[\alpha] \widetilde{\star}[\beta]=[\alpha \star \beta]
$$

(where $[\alpha]$ denotes the class of equivalence of $\alpha \in \operatorname{Mon}^{\uparrow}(F)$ ). Then $\widetilde{\star}$ is well-defined and it is an associative operation on $\frac{\operatorname{Mon}^{\uparrow}(F)}{\sim}$. 
Proof. Let $\alpha_{1}, \alpha_{2}, \beta_{1}, \beta_{2} \in \operatorname{Mon}^{\uparrow}(F)$ be such that $\left[\alpha_{1}\right]=\left[\alpha_{2}\right]$ and $\left[\beta_{1}\right]=\left[\beta_{2}\right]$. Then $\alpha_{1} \sim \alpha_{2}$ and $\beta_{1} \sim \beta_{2}$. By item 1 of Theorem 5.12, $\alpha_{1} \star \beta_{1} \sim \alpha_{2} \star \beta_{2}$, which means that $\left[\alpha_{1} \star \beta_{1}\right]=\left[\alpha_{2} \star \beta_{2}\right]$. Hence $\left[\alpha_{1}\right] \widetilde{\star}\left[\beta_{1}\right]=\left[\alpha_{2}\right] \widetilde{\star}\left[\beta_{2}\right]$ and the operation $\widetilde{\star}$ is well-defined. Furthermore, let $[\alpha],[\beta],[\gamma] \in \frac{\operatorname{Mon}^{\uparrow}(F)}{\sim}$ be three classes of equivalence. By item 2 of Theorem 5.12, $(\alpha \star \beta) \star \gamma \sim \alpha \star(\beta \star \gamma)$, which means that $[(\alpha \star \beta) \star \gamma]=[\alpha \star(\beta \star \gamma)]$. As a consequence,

$$
\begin{aligned}
([\alpha] \widetilde{\star}[\beta]) \widetilde{\star}[\gamma] & =[\alpha \star \beta] \widetilde{\star}[\gamma]=[(\alpha \star \beta) \star \gamma]=[\alpha \star(\beta \star \gamma)]= \\
& =[\alpha] \approx[\beta \star \gamma]=[\alpha] \star([\beta] \star[\gamma]),
\end{aligned}
$$

and $\widetilde{\star}$ is associative.

\section{Idempotency and averaging behaviour for $\alpha$-monotone fusion functions}

In this section, we analyze the notions of idempotence and averaging behaviour of a fusion function in terms of $\alpha$-monotonicity. We start proving a sufficient condition for a fusion function to be idempotent.

Proposition 6.1. Given a fusion function $F:[0,1]^{n} \rightarrow[0,1]$, assume that there are $\lambda_{1}, \lambda_{2} \geq 0$ such that

$$
\min (\mathbf{x})-\lambda_{1} \max _{1 \leq i, j \leq n}\left\{\left|x_{i}-x_{j}\right|\right\} \leq F(\mathbf{x}) \leq \max (\mathbf{x})+\lambda_{2} \max _{1 \leq i, j \leq n}\left\{\left|x_{i}-x_{j}\right|\right\}
$$

for all $\mathbf{x} \in[0,1]^{n}$. Then $F$ is idempotent.

Proof. It is enough to take $\mathbf{x}=(t, \ldots, t)$ for $t \in[0,1]$.

Now we present a first relationship between idempotency and $\alpha$-monotonicity. For simplicity, given $t \in[0,1]$, we denote $\mathbf{t}=(t, t, \ldots, t) \in[0,1]^{n}$.

Theorem 6.2. Let $\alpha: I_{\alpha} \rightarrow \mathbb{R}^{n}$ be a curve continuous at $t=0$ and let $F:[0,1]^{n} \rightarrow$ $[0,1]$ be an $\alpha$-increasing fusion function. Let us consider the following assertions.

(a) $F$ is idempotent.

(b) For every $t \in[0,1]$ and every $r, s \in I_{\alpha}$ such that $\left(C_{r, s}^{\mathbf{t}, \alpha}\right)$ holds, we have that

$$
F(\mathbf{t}-\alpha(r)) \leq t \leq F(\mathbf{t}+\alpha(s))
$$

(c) For every $\varepsilon>0$ we have that

$$
F(\mathbf{t}-\alpha(r)) \leq t \leq F(\mathbf{t}+\alpha(s))
$$

for every $t \in[0,1]$ and every $r, s \in I_{\alpha}$ for which $\left(C_{r, s}^{\mathbf{t}, \alpha}\right)$ holds and $\max \left(\|\alpha(r)\|_{1},\|\alpha(s)\|_{1}\right) \leq \varepsilon$.

(d) There exists $\varepsilon \in] 0,1[$ such that

$$
F(\mathbf{t}-\alpha(r)) \leq t \leq F(\mathbf{t}+\alpha(s))
$$


for every $t \in[0,1]$ and every $r, s \in I_{\alpha}$ for which $\left(C_{r, s}^{\mathbf{t}, \alpha}\right)$ holds and $\max \left(\|\alpha(r)\|_{1},\|\alpha(s)\|_{1}\right) \leq \varepsilon$.

(e) For all $t \in] 0,1\left[\right.$ there exists $\varepsilon_{t}>0$ such that

$$
F(\mathbf{t}-\alpha(r)) \leq t \leq F(\mathbf{t}+\alpha(s))
$$

for all $r, s \in I_{\alpha}$ for which $\left(C_{r, s}^{\mathbf{t}, \alpha}\right)$ holds and $\max \left(\|\alpha(r)\|_{1},\|\alpha(s)\|_{1}\right) \leq \varepsilon_{t}$.

Then $(a) \Rightarrow(b) \Rightarrow(c) \Rightarrow(d) \Rightarrow(e)$.

In addition to this, if $F$ is continuous on $\mathbf{0}$ and on, $\mathbf{1}$, then the previous assertions are equivalent.

Proof. $(a) \Rightarrow(b)$ follows from Theorem 4.9 , and $(b) \Rightarrow(c) \Rightarrow(d) \Rightarrow(e)$ are straightforward exercises. Suppose that $F$ is continuous on $\mathbf{0}$ and on, $\mathbf{1}$, and let us show that $(e) \Rightarrow(a)$. Let $t \in] 0,1[$ be arbitrary and we have to prove that $F(\mathbf{t})=t$. Using $r=s=0$ on $(e)$, we deduce that $F(\mathbf{t})=t$. And as $F$ is continuous on $\mathbf{0}$ and on , $\mathbf{1}$, then also $F(\mathbf{0})=0$ and $F(\mathbf{1})=1$, so $F$ is idempotent.

Next, we are going to characterize the class of averaging fusion functions which are $\alpha$-increasing.

Theorem 6.3. Let $\alpha: I_{\alpha} \rightarrow \mathbb{R}^{n}$ be a curve and let $F:[0,1]^{n} \rightarrow[0,1]$ be an $\alpha$ increasing fusion function. Then the following statements are equivalent.

(1) $F$ is averaging.

(2) For all $\mathbf{x} \in[0,1]^{n}$ and all $r, s \in I_{\alpha}$ such that $\left(C_{r, s}^{\mathbf{x}, \alpha}\right)$ holds we have that

$$
\max \{\min (\mathbf{x}), \min (\mathbf{x}-\alpha(r))\} \leq F(\mathbf{x}) \leq \min \{\max (\mathbf{x}), \max (\mathbf{x}+\alpha(s))\} .
$$

Proof. The fact that (2) implies (1) is straightforward just taking $r=s=0$. To see that (1) implies (2), assume that $F$ is averaging and let $\mathbf{x} \in[0,1]^{n}$ and $r, s \in[0, \theta[$ be such that $\left(C_{r, s}^{\mathbf{x}, \alpha}\right)$ holds. On the one hand, as $F$ is averaging,

$$
\min (\mathbf{x}) \leq F(\mathbf{x}) \leq \max (\mathbf{x}) .
$$

On the other hand, from Theorem 4.9, we have that

$$
\min (\mathbf{x}-\alpha(r)) \leq F(\mathbf{x}-\alpha(r)) \leq F(\mathbf{x}) \leq F(\mathbf{x}+\alpha(s)) \leq \max (\mathbf{x}+\alpha(s)) .
$$

By combining both inequalities we conclude that (9) is fulfilled.

Remark 6.4. The condition

$$
\min (\mathbf{x}) \leq F(\mathbf{x}) \leq \max (\mathbf{x})
$$

is very restrictive on small neighbourhoods of the points $(0, \ldots, 0)$ and $(1, \ldots, 1)$. But, at the same time, it is quite weak far from these two points, and it can even be empty for points $\mathbf{x}$ such that $\min (\mathbf{x})=0$ and $\max (\mathbf{x})=1$. As a consequence, the condition provided by inequalities (9) is restrictive for an $\alpha$-increasing function when all components of $\alpha$ are negative (and when all component of $\alpha$ are positive for an $\alpha$-decreasing function). 
Corollary 6.5. Let $\alpha: I_{\alpha} \rightarrow \mathbb{R}^{n}$ be a curve and let $F:[0,1]^{n} \rightarrow[0,1]$ be an $\alpha$ increasing fusion function. If $\alpha_{i}(s) \leq 0$ for all $s \in I_{\alpha}$ and all $i \in\{1, \ldots, n\}$, then the following statements are equivalent.

(1) $F$ is averaging.

(2) For all $\mathbf{x} \in[0,1]^{n}$ and all $r, s \in I_{\alpha}$ such that $\left(C_{r, s}^{\mathbf{x}, \alpha}\right)$ holds we have that

$$
\min _{1 \leq i \leq n}\left\{x_{i}+\left|\alpha_{i}(s)\right|\right\} \leq F(\mathbf{x}) \leq \max _{1 \leq i \leq n}\left\{x_{i}-\left|\alpha_{i}(s)\right|\right\} .
$$

Proof. It is enough to observe that, for all $\mathbf{x} \in[0,1]^{n}$ and $r, s \in I_{\alpha}$ such that $\left(C_{r, s}^{\mathbf{x}, \alpha}\right)$ holds, we have that

$$
\min (\mathbf{x}-\alpha(r))=\min _{1 \leq i \leq n}\left\{x_{i}-\alpha_{i}(s)\right\}=\min _{1 \leq i \leq n}\left\{x_{i}+\left|\alpha_{i}(s)\right|\right\} \geq \min (\mathbf{x})
$$

and

$$
\max (\mathbf{x}+\alpha(s))=\max _{1 \leq i \leq n}\left\{x_{i}+\alpha_{i}(s)\right\}=\max _{1 \leq i \leq n}\left\{x_{i}-\left|\alpha_{i}(s)\right|\right\} \leq \max (\mathbf{x}) .
$$

The result follows from Theorem 6.3.

In other words, negative components of $\alpha$ are useful to restrict or control the class of $\alpha$-increasing averaging fusion functions.

Example 6.6. In order to consider a more elaborate example, let $\mathbf{y}=$ $\left.\left(y_{1}, y_{2}, \ldots, y_{n}\right) \in\right] 0, \infty\left[{ }^{n}\right.$ and let $\left.G=\left(g_{1}, \ldots, g_{n}\right):[0,1]^{n} \rightarrow\right] 0, \infty\left[{ }^{n}\right.$. Let us define $F:[0,1]^{n} \rightarrow[0, \infty[$ by

$$
F(\mathbf{x})= \begin{cases}\frac{G(\mathbf{x}) \cdot \mathbf{x}}{\mathbf{y} \cdot \mathbf{x}}, & \text { if } \mathbf{x} \neq \mathbf{0} \\ 0, & \text { otherwise }\end{cases}
$$

Then $F$ is well defined. Moreover, $F$ is a fusion function if and only if $(\mathbf{y}-G(\mathbf{x})) \cdot \mathbf{x} \geq 0$ for all $\mathbf{x} \in[0,1]^{n}$. In particular, note that if $G$ is bounded (for instance, if it is continuous), there exists $\mathbf{y}=(y, \ldots, y) \in \mathbb{R}^{n}$ with $y>0$ and such that $F$ is a fusion function.

So let us assume that $F$ is a fusion function and denote $\mathbf{e}_{i}=\left(0, \ldots, 0, \frac{1}{i}, 0, \ldots 0\right) \in$ $[0,1]^{n}$. Then

$$
1 \geq F\left(\mathbf{e}_{i}\right)=\frac{G\left(\mathbf{e}_{i}\right) \cdot \mathbf{e}_{i}}{\mathbf{y} \cdot \mathbf{e}_{i}}=\frac{g_{i}\left(\mathbf{e}_{i}\right)}{y_{i}}
$$

so it follows that $g_{i}\left(\mathbf{e}_{\mathbf{i}}\right) \leq y_{i}$ for every $i \in\{1, \ldots, n\}$. Furthermore, $F$ is idempotent if and only if

$$
\|G(t, \ldots, t)\|_{1}=t\|\mathbf{y}\|_{1}
$$


for every $t \in] 0,1]$, since

$$
F(t, \ldots, t)=\frac{\sum_{i=1}^{n} g_{i}(t, \ldots, t) t}{\sum_{i=1}^{n} y_{i} t}=\frac{\sum_{i=1}^{n} g_{i}(t, \ldots, t)}{\sum_{i=1}^{n} y_{i}}=\frac{\|G(t, \ldots, t)\|_{1}}{\|\mathbf{y}\|_{1}}
$$

In this case, we have

$$
g_{n}(t, \ldots, t)=t\|\mathbf{y}\|_{1}-\sum_{i=1}^{n-1} g_{i}(t, \ldots, t)
$$

for all $t \in] 0,1]$. Furthermore, if

$$
\min \{(G(\mathbf{x})-\min (\mathbf{x}) \mathbf{y}) \cdot \mathbf{x},(\max (\mathbf{x}) \mathbf{y}-G(\mathbf{x})) \cdot \mathbf{x}\} \geq 0
$$

for every $\mathbf{x}, \mathbf{y} \in[0,1]^{n} \backslash\{\mathbf{0}\}$, then $F$ is an averaging function.

Example 6.7. Let $A \in \mathcal{M}_{n}(\mathbb{R})$ be a symmetric, positive definite matrix of order $n$ and let $\mathbf{y} \in] 0, \infty\left[{ }^{n}\right.$. Let us define $F:[0,1]^{n} \rightarrow[0, \infty[$ by

$$
F(\mathbf{x})= \begin{cases}\frac{\mathbf{x}^{T} \cdot A \cdot \mathbf{x}}{\mathbf{y} \cdot \mathbf{x}}, & \text { if } \mathbf{x} \neq \mathbf{0}, \\ 0, & \text { otherwise. }\end{cases}
$$

If $\mathbf{x}^{T} \cdot A \cdot \mathbf{x} \leq \mathbf{y} \cdot \mathbf{x}$ for every $\mathbf{x}$, then $F$ is a fusion function. In this setting, if $\alpha:\left[0, \theta\left[\rightarrow \mathbb{R}^{n}\right.\right.$ is a curve such that $\mathbf{y} \cdot \alpha(t)>0$ for every $t \in] 0, \theta[$, then $F$ is $\alpha$-increasing if and only if

$$
\frac{F(\mathbf{x})-F(\alpha(t))}{2} \leq \frac{\mathbf{x}^{T} \cdot A \cdot \alpha(t)}{\mathbf{y} \cdot \alpha(t)}
$$

for all $\mathbf{x} \in[0,1]^{n}$ and all $\left.t \in\right] 0, \theta\left[\right.$ such that $\mathbf{x}+\alpha(t) \in[0,1]^{n} \backslash\{\mathbf{0}\}$.

\section{Conclusion and future research}

In this work we have introduced the notion of curve-based monotonicity as an extension of both the usual notion of monotonicity as well as its recent generalizations, weak and directional monotonicity. This study is relevant since, as discussed in the examples, there are examples of functions which are interesting from the point of view of the applications, but which are not monotone or directionally monotone. In future works we will further develop theoretically this concept, and we will analyze its possible applications. In particular, the consideration of possible applications in image processing and, more specifically, in edge detection would be of interest. In addition, taking into account the excellent results that pre-aggregation functions have provided in classification problems, we will look for the use of the new notion in this field. 


\section{Funding}

This work is supported by the research group FQM268 of Junta de Andalucia, by the projects TIN2017-89517-P and TIN2016-77356-P (AEI/FEDER, UE), by the the Slovak Scientific Grant Agency VEGA no. 1/0093/17 Identification of risk factors and their impact on products of the insurance and savings schemes, by Slovak grant APVV14-0013, and by Czech Project LQ1602 "IT4Innovations excellence in science".

\section{References}

Beliakov, G. 2005. "Monotonicity preserving approximation of multivariate scattered data." BIT numerical mathematics 45 (4): 653-677.

Beliakov, G., H. Bustince, T. Calvo. 2016. A practical guide to averaging functions Series: Studies in Fuzziness and Soft Computing. Heidelberg: Springer.

Beliakov, G., T. Calvo, T. Wilkin. 2014. "Three types of monotonicity of averaging functions." Knowledge-Based Systems 72: 114-122.

Beliakov, G., T. Calvo, T. Wilkin. 2015. "On the weak monotonicity of Gini means and other mixture functions." Information Sciences 300: 70-84.

Bustince, H., E. Barrenechea, T. Calvo, S. James, G. Beliakov. 2014. "Consensus in multiexpert decision making problems using penalty functions defined over a Cartesian product of lattices." Information Fusion 17: 56-64.

Bustince, H., E. Barrenechea, M. Pagola. 2006. "Restricted equivalence functions." Fuzzy Sets and Systems 157: 2333-2346.

Bustince, H., E. Barrenechea, M. Sesma-Sara, J. Lafuente, G.P. Dimuro, R. Mesiar and A. Kolesárová. 2018. "Ordered Directionally Monotone Functions: Justification and Application." IEEE Transactions on Fuzzy Systems 26 (4): 2237-2250.

Bustince, H., P. Burillo, F. Soria. 2003. "Automorphisms, negations and implication operators." Fuzzy Sets and Systems 134: 209-229.

Bustince, H., J. Fernández, A. Kolesárová, R. Mesiar. 2015. "Directional monotonicity of fusion functions." European Journal of Operational Research 244 (1): 300-308.

Bustince, H., M. Pagola, E. Barrenechea. 2007. "Construction of fuzzy indices from fuzzy DI-subsethood measures: Application to the global comparison of images." Information Sciences 177 (3): 906-929.

Bustince, H., M. Pagola, R. Mesiar, E. Hüllermeier, F. Herrera. 2012. "Grouping, Overlap, and Generalized Bientropic Functions for Fuzzy Modeling of Pairwise Comparisons." IEEE Transactions on Fuzzy Systems 20: 405-415.

Calvo, T., A. Kolesárová, M. Komorníková, R. Mesiar. 2002. "Aggregation Operators: Properties, Classes and Construction Methods." In Aggregation Operators. New Trends and Applications, edited by T. Calvo, G. Mayor and R. Mesiar, 3-104. Heidelberg: PhysicaVerlag.

Gómez, D., J. Montero. 2004. "A discussion on aggregation operators." Kybernetika 40: 107120.

Grabisch, M., J.-L. Marichal, R. Mesiar, E. Pap. 2009. Aggregation Functions. Cambridge: Cambridge University Press.

Hall, D.K., G.A. Riggs, V.V. Salomonson. 1995. "Development of methods for mapping global snow cobres using moderate resolution imaging spectroradiometer data." Remote Sensing of Environment 54: 127-140.

Jurio, A., H. Bustince, M. Pagola, A. Pradera, R.R. Yager. 2013. "Some properties of overlap and grouping functions and their application to image thresholding." Fuzzy Sets and Systems 229: 69-90.

Lucca, G., J. Sanz, G. Pereira Dimuro, B. Bedregal, R. Mesiar, A. Kolesárová, H. Bustince. 2016. "Pre-aggregation functions: construction and an application." IEEE Transactions on 
Fuzzy Systems 24: 260-272.

Melin, P., D. Bravo and O. Castillo. 2008. "Fingerprint recognition using the fuzzy Sugeno integral for response integration in modular neural networks." International Journal of General Systems 37 (4): 499-515.

Mesiar, R., A. Kolesárová and A. Stupňanová. 2018. "Quo vadis aggregation?” International Journal of General Systems 47 (2): 97-117.

Sesma-Sara, M., L. De Miguel, M. Pagola, A. Burusco, R. Mesiar and H. Bustince. 2018. "New measures for comparing matrices and their application to image processing." Applied Mathematical Modelling 61: 498-520.

Sesma-Sara, M., J. Lafuente, A. Roldán, R. Mesiar, H. Bustince. "Strengthened ordered directionally monotone functions. Links between the different notions of monotonicity." Fuzzy Sets and Systems In press.

Soria-Frisch, A. 2009. "Optical-SAR fusion based snow detection." Technical Report TN00186, Starlab Barcelona

Storvold, R., E. Malnes. 2004. "Snow covered area retrieval using envisat asar wideswath in mountainous areas." In: Proc. IEEE International Geoscience and Remote Sensing Symposium, IGARSS 2004: 1845-1848.

$\mathrm{Su}, \mathrm{Z} ., \mathrm{G}$. Xia and M. Chen. 2011. "Some induced intuitionistic fuzzy aggregation operators applied to multi-attribute group decision making." International Journal of General Systems 40 (8): 805-835.

Wilkin, T., G. Beliakov. 2015. "Weakly Monotonic Averaging Functions." International Journal of Intelligent Systems 30 (2): 144-169.

Ye, J. "Multicriteria decision-making method using the correlation coefficient under singlevalued neutrosophic environment." International Journal of General Systems 42 (4): 386394. 